\title{
Multivariable $(\varphi, \Gamma)$-modules and products of Galois groups
}

\author{
Gergely Zábrádi *
}

14th March 2016

\begin{abstract}
We show that the category of continuous representations of the $d$ th direct power of the absolute Galois group of $\mathbb{Q}_{p}$ on finite dimensional $\mathbb{F}_{p}$-vector spaces (resp. finitely generated $\mathbb{Z}_{p}$-modules, resp. finite dimensional $\mathbb{Q}_{p}$-vector spaces) is equivalent to the category of étale $(\varphi, \Gamma)$-modules over a $d$-variable Laurent-series ring over $\mathbb{F}_{p}$ (resp. over $\mathbb{Z}_{p}$, resp. over $\left.\mathbb{Q}_{p}\right)$.
\end{abstract}

\section{Introduction}

This note serves as a complement to the work [11] where we relate multivariable $(\varphi, \Gamma)$ modules to smooth modulo $p^{n}$ representations of a split reductive group $G$ over $\mathbb{Q}_{p}$. The goal here is to show that the category of $d$-variable $(\varphi, \Gamma)$-modules is equivalent to the category of representations of the $d$ th direct power of the absolute Galois group of $\mathbb{Q}_{p}$.

Let $K$ be a finite extension of $\mathbb{Q}_{p}$ with ring of integers $\mathcal{O}_{K}$, prime element $\varpi$, and residue field $\kappa$. For a finite set $\Delta$ let $G_{\mathbb{Q}_{p}, \Delta}:=\prod_{\alpha \in \Delta} \operatorname{Gal}\left(\overline{\mathbb{Q}_{p}} / \mathbb{Q}_{p}\right)$ denote the direct power of the absolute Galois group of $\mathbb{Q}_{p}$ indexed by $\Delta$. We denote by $\operatorname{Rep}{ }_{\kappa}\left(G_{\mathbb{Q}_{p}, \Delta}\right)\left(\operatorname{resp}\right.$. by $\operatorname{Rep}_{\mathcal{O}_{K}}\left(G_{\mathbb{Q}_{p}, \Delta}\right)$, resp. by $\left.\operatorname{Rep}_{K}\left(G_{\mathbb{Q}_{p}, \Delta}\right)\right)$ the category of continuous representations of the profinite group $G_{\mathbb{Q}_{p}, \Delta}$ on finite dimensional $\kappa$-vector spaces (resp. finitely generated $\mathcal{O}_{K}$-modules, resp. finite dimensional $K$-vector spaces). On the other hand, for independent commuting variables $X_{\alpha}(\alpha \in \Delta)$ we put

$$
\begin{aligned}
E_{\Delta, \kappa} & :=\kappa\left[\left[X_{\alpha} \mid \alpha \in \Delta\right]\right]\left[X_{\alpha}^{-1} \mid \alpha \in \Delta\right] \\
\mathcal{O}_{\mathcal{E}_{\Delta, K}} & :=\underbrace{\lim }_{h}\left(\mathcal{O}_{K} / \varpi^{h}\left[\left[X_{\alpha} \mid \alpha \in \Delta\right]\right]\left[X_{\alpha}^{-1} \mid \alpha \in \Delta\right]\right) \\
\mathcal{E}_{\Delta, K} & :=\mathcal{O}_{\mathcal{E}_{\Delta, K}}\left[p^{-1}\right] .
\end{aligned}
$$

Moreover, for each element $\alpha \in \Delta$ we have the partial Frobenius $\varphi_{\alpha}$, and group $\Gamma_{\alpha} \cong$ $\operatorname{Gal}\left(\mathbb{Q}_{p}\left(\mu_{p^{\infty}}\right) / \mathbb{Q}_{p}\right)$ acting on the variable $X_{\alpha}$ in the usual way and commuting with the other

*This research was supported by a Hungarian OTKA Research grant K-100291 and by the János Bolyai Scholarship of the Hungarian Academy of Sciences. I would like to thank the Arithmetic Geometry and Number Theory group of the University of Duisburg-Essen, campus Essen, for its hospitality and for financial support from SFB TR45 where parts of this paper was written. 
variables $X_{\beta}(\beta \in \Delta \backslash\{\alpha\})$ in the above rings. A $\left(\varphi_{\Delta}, \Gamma_{\Delta}\right)$-module over $E_{\Delta, \kappa}$ (resp. over $\mathcal{O}_{\mathcal{E}_{\Delta, K}}$, resp. over $\mathcal{E}_{\Delta, K}$ ) is a finitely generated $E_{\Delta, \kappa}$-module (resp. $\mathcal{O}_{\mathcal{E}_{\Delta, K}}$-module, resp. $\mathcal{E}_{\Delta, K}$-module) $D$ together with commuting semilinear actions of the operators $\varphi_{\alpha}$ and groups $\Gamma_{\alpha}(\alpha \in \Delta)$. In case the coefficient ring is $E_{\Delta, \kappa}$ or $\mathcal{O}_{\mathcal{E}_{\Delta, K}}$, we say that $D$ is étale if the map id $\otimes \varphi_{\alpha}: \varphi_{\alpha}^{*} D \rightarrow D$ is an isomorphism for all $\alpha \in \Delta$. For the coefficient ring $\mathcal{E}_{\Delta, K}$ we require the stronger assumption for the étale property that $D$ comes from an étale $\left(\varphi_{\Delta}, \Gamma_{\Delta}\right)$-module over $\mathcal{O}_{\mathcal{E}_{\Delta, K}}$ by inverting $p$. The main result of the paper is that $\operatorname{Rep}_{\kappa}\left(G_{\mathbb{Q}_{p}, \Delta}\right)\left(\operatorname{resp} \cdot \operatorname{Rep}_{\mathcal{O}_{K}}\left(G_{\mathbb{Q}_{p}, \Delta}\right)\right.$, resp. $\left.\operatorname{Rep}_{K}\left(G_{\mathbb{Q}_{p}, \Delta}\right)\right)$ is equivalent to the category of étale $\left(\varphi_{\Delta}, \Gamma_{\Delta}\right)$-modules over $E_{\Delta, \kappa}$ (resp. over $\mathcal{O}_{\mathcal{E}_{\Delta, K}}$, resp. over $\left.\mathcal{E}_{\Delta, K}\right)$

Passing from the Galois side to $\left(\varphi_{\Delta}, \Gamma_{\Delta}\right)$-modules is rather straightforward. One constructs a big ring $E_{\Delta}^{s e p}$ as an inductive limit of completed tensor products of finite separable extensions $E_{\alpha}^{\prime}$ of $E_{\alpha}=\mathbb{F}_{p}\left(\left(X_{\alpha}\right)\right)(\alpha \in \Delta)$ over which the action of $H_{\mathbb{Q}_{p}, \Delta}=\operatorname{Ker}\left(G_{\mathbb{Q}_{p}, \Delta} \rightarrow \prod_{\alpha \in \Delta} \Gamma_{\Delta}\right)$ trivializes. The other direction is more involved. In order to trivialize the action of the partial Frobenii $\varphi_{\alpha}(\alpha \in \Delta)$ using induction, the main step is to find a lattice $D_{\bar{\alpha}}^{+*}$ integral in the variable $X_{\alpha}$ for some fixed $\alpha \in \Delta$ which is an étale $\left(\varphi_{\Delta \backslash\{\alpha\}}, \Gamma_{\Delta \backslash\{\alpha\}}\right)$-module over the ring $\mathbb{F}_{p}\left[\left[X_{\beta} \mid \beta \in \Delta\right]\right]\left[X_{\beta}^{-1} \mid \beta \in \Delta \backslash\{\alpha\}\right]$. This uses the ideas of Colmez [3] constructing lattices $D^{+}$and $D^{++}$in usual $(\varphi, \Gamma)$-modules.

We remark here that Scholze [7] recently realized $G_{\mathbb{Q}_{p}, \Delta}$ (using Drinfeld's Lemma for diamonds) as a geometric fundamental group $\pi_{1}\left(\left(\operatorname{Spd} \mathbb{Q}_{p}\right)^{|\Delta|} /\right.$ p.Fr.) of the diamond $\left(\operatorname{Spd} \mathbb{Q}_{p}\right)^{|\Delta|}$ modulo the partial Frobenii $\varphi_{\beta}(\beta \in \Delta \backslash\{\alpha\})$ for some fixed $\alpha \in \Delta$ : one can endow $E_{\Delta}^{+}=\mathbb{F}_{p}\left[\left[X_{\alpha} \mid \alpha \in \Delta\right]\right]$ with its natural compact topology, and look at the subset of its adic spectrum Spa $E_{\Delta}^{+}$where all $X_{\alpha}(\alpha \in \Delta)$ are invertible. This defines an analytic adic space over $\mathbb{F}_{p}$, whose perfection modulo the action of all $\Gamma_{\alpha}$ 's is a model for $\left(\operatorname{Spd} \mathbb{Q}_{p}\right)^{d}$. Thus, after taking the action modulo partial Frobenii $\varphi_{\beta}(\beta \in \Delta \backslash\{\alpha\}$ for some fixed $\alpha \in \Delta)$, the fundamental group will be $G_{\mathbb{Q}_{p}, \Delta}$. Now, quite generally étale local systems on diamonds are equivalent to $\varphi$-modules. This introduces the last missing Frobenius, and one ends up with an equivalence between representations of $G_{\mathbb{Q}_{p}, \Delta}$, and some sheaf of modules with $\Gamma_{\Delta}$-action and commuting actions of $\varphi_{\alpha}$ for all $\alpha \in \Delta$. However, this will not produce an actual module over a ring, but a sheaf of modules over a sheaf of rings. One can perhaps deduce the result of this paper along these lines, but that would require some further nontrivial input (replacing the above method of finding a lattice $D_{\bar{\alpha}}^{+*}$ ).

\subsection{Acknowledgements}

I would like to thank Christophe Breuil, Elmar Große-Klönne, Kiran Kedlaya, and Vytas Paškūnas for useful discussions on the topic. I would like to thank Peter Scholze for clarifying the relation of this work to his theory of realizing $G_{\mathbb{Q}_{p}, \Delta}$ as the étale fundamental group of a diamond.

\section{Algebraic properties of multivariable $(\varphi, \Gamma)$-modules}

\subsection{Definition and projectivity}

For a finite set $\Delta$ (which is the set of simple roots of $G$ in [11]) consider the Laurent series $\operatorname{ring} E_{\Delta}:=E_{\Delta}^{+}\left[X_{\Delta}^{-1}\right]$ where $E_{\Delta}^{+}:=\mathbb{F}_{p}\left[\left[X_{\alpha} \mid \alpha \in \Delta\right]\right]$ and $X_{\Delta}:=\prod_{\alpha \in \Delta} X_{\alpha} \in E_{\Delta}^{+}$. $E_{\Delta}^{+}$is a 
regular noehterian local ring of global dimension $|\Delta|$, therefore $E_{\Delta}$ is a regular noetherian ring of global dimension $|\Delta|-1$. For each index $\alpha$ we define the action of the partial Frobenius $\varphi_{\alpha}$ and of the group $\Gamma_{\alpha}$ with $\chi_{\alpha}: \Gamma_{\alpha} \stackrel{\sim}{\rightarrow} \mathbb{Z}_{p}^{\times}$on $E_{\Delta}$ as

$$
\begin{aligned}
\varphi_{\alpha}\left(X_{\beta}\right) & := \begin{cases}X_{\beta} & \text { if } \beta \in \Delta \backslash\{\alpha\} \\
\left(X_{\alpha}+1\right)^{p}-1=X_{\alpha}^{p} & \text { if } \beta=\alpha\end{cases} \\
\gamma_{\alpha}\left(X_{\beta}\right) & := \begin{cases}X_{\beta} & \text { if } \beta \in \Delta \backslash\{\alpha\} \\
\left(X_{\alpha}+1\right)^{\chi_{\alpha}\left(\gamma_{\alpha}\right)}-1 & \text { if } \beta=\alpha\end{cases}
\end{aligned}
$$

for all $\gamma_{\alpha} \in \Gamma_{\alpha}$ extending the above formulas to continuous ring endomorphisms of $E_{\Delta}$ in the obvious way. By an étale $\left(\varphi_{\Delta}, \Gamma_{\Delta}\right)$-module over $E_{\Delta}$ we mean a (unless otherwise mentioned) finitely generated module $D$ over $E_{\Delta}$ together with a semilinear action of the (commutative) monoid $T_{+, \Delta}:=\prod_{\alpha \in \Delta} \varphi_{\alpha}^{\mathbb{N}} \Gamma_{\alpha}$ (also denote by $\varphi_{t}$ the action of $\varphi_{t} \in T_{+, \Delta}$ ) such that the maps

$$
\text { id } \otimes \varphi_{t}: \varphi_{t}^{*} D:=E_{\Delta} \otimes_{E_{\Delta}, \varphi_{t}} D \rightarrow D
$$

are isomorphisms for all elements $\varphi_{t} \in T_{+, \Delta}$. Here we put $\Gamma_{\Delta}:=\prod_{\alpha \in \Delta} \Gamma_{\alpha}$. We denote by $\mathcal{D}^{e t}\left(\varphi_{\Delta}, \Gamma_{\Delta}, E_{\Delta}\right)$ the category of étale $\left(\varphi_{\Delta}, \Gamma_{\Delta}\right)$-modules over $E_{\Delta}$.

The category $\mathcal{D}^{e t}\left(\varphi_{\Delta}, \Gamma_{\Delta}, E_{\Delta}\right)$ has the structure of a neutral Tannakian category: For two objects $D_{1}$ and $D_{2}$ the tensor product $D_{1} \otimes_{E_{\Delta}} D_{2}$ is an étale $T_{+, \Delta}$-module with the action $\varphi_{t}\left(d_{1} \otimes d_{2}\right):=\varphi_{t}\left(d_{1}\right) \otimes \varphi_{t}\left(d_{2}\right)$ for $\varphi_{t} \in T_{+, \Delta}, d_{i} \in D_{i}(i=1,2)$. Moreover, since $E_{\Delta}$ is a free module over itself via $\varphi_{t}$, putting $(\cdot)^{*}:=\operatorname{Hom}_{E_{\Delta}}\left(\cdot, E_{\Delta}\right)$ we have an identification $\left(\varphi_{t}^{*} D\right)^{*} \cong$ $\varphi_{t}^{*}\left(D^{*}\right)$. So the isomorphism id $\otimes \varphi_{t}: \varphi_{t}^{*} D \rightarrow D$ dualizes to an isomorphism $D^{*} \rightarrow \varphi_{t}^{*}\left(D^{*}\right)$. The inverse of this isomorphism (for all $\varphi_{t} \in T_{+, \Delta}$ ) equips $D^{*}$ with the structure of an étale $T_{+, \Delta}$-module.

Lemma 2.1. There exists a $\Gamma_{\Delta}$-equivariant injective resolution of $E_{\Delta}^{+}$as a module over itself. Proof. Consider the Cousin complex (see IV.2 in [6])

$$
0 \rightarrow E_{\Delta} \rightarrow E_{\Delta,(0)} \rightarrow \cdots \rightarrow \bigoplus_{\mathfrak{p} \in \operatorname{Spec}\left(E_{\Delta}\right), \operatorname{codim} \mathfrak{p}=r} J(\mathfrak{p}) \rightarrow \ldots
$$

where $J(\mathfrak{p})$ is the injective envelope of the residue field $\kappa(\mathfrak{p})$ as a module over the local ring $E_{\Delta, \mathfrak{p}}$. This is a $\Gamma_{\Delta}$-equivariant injective resolution since the action of $\Gamma_{\Delta}$ on $\operatorname{Spec}\left(E_{\Delta}\right)$ respects the codimension.

Proposition 2.2. Any object $D$ in $\mathcal{D}^{e t}\left(\varphi_{\Delta}, \Gamma_{\Delta}, E_{\Delta}\right)$ is a projective module over $E_{\Delta}$.

Proof. Since $E_{\Delta}$ has finite global dimension, let $n$ be the projective dimension of $D$. Then by Lemma 4.1.6 in [9] we have $\operatorname{Ext}^{i}(D, M)=0$ for all $i>n$ and $E_{\Delta}$-module $M$ and there exists an $R$-module $M_{0}$ with $\operatorname{Ext}^{n}\left(D, M_{0}\right) \neq 0$. By the long exact sequence of Ext and choosing an onto module homomorphism $F \rightarrow M_{0}$ from a free module $F$ we find that $\operatorname{Ext}^{n}(D, F) \neq 0$ whence $\operatorname{Ext}^{n}\left(D, E_{\Delta}\right) \neq 0$. However, $\operatorname{Ext}^{n}\left(D, E_{\Delta}\right)$ is a finitely generated torsion $E_{\Delta}$-module for $n>0$ admitting a semilinear action of $\Gamma_{\Delta}$. Therefore the global annihilator of $\operatorname{Ext}^{n}\left(D, E_{\Delta}\right)$ in $E_{\Delta}$ is a nonzero $\Gamma_{\Delta}$-invariant ideal in $E_{\Delta}$ hence equals $E_{\Delta}$ by Lemma 2.1 in [11]. So $n=0$ and $D$ is projective. 
Lemma 2.3. We have $K_{0}\left(E_{\Delta}\right) \cong \mathbb{Z}$, ie. any finitely generated projective module over $E_{\Delta}$ is stably free.

Proof. $E_{\Delta}^{+} \cong \mathbb{F}_{p}\left[\left[X_{\alpha} \mid \alpha \in \Delta\right]\right]$ is a regular local ring, so it has finite global dimension and $K_{0}\left(E_{\Delta}^{+}\right) \cong G_{0}\left(E_{\Delta}^{+}\right) \cong \mathbb{Z}$ (Thm. II.7.8 in [10]). Therefore the localization $E_{\Delta}=E_{\Delta}^{+}\left[X_{\Delta}^{-1}\right]$ also has finite global dimension whence we have $K_{0}\left(E_{\Delta}\right) \cong G_{0}\left(E_{\Delta}\right)$. The statement follows noting that the map $G_{0}\left(E_{\Delta}^{+}\right) \rightarrow G_{0}\left(E_{\Delta}\right)$ is onto by the localization exact sequence of algebraic $K$-theory (Thm. II.6.4 in [10]).

Remark. I am not aware of the analogue of the Theorem of Quillen and Suslin on the freeness of projective modules over $E_{\Delta}$. However, using the equivalence of categories of $\mathcal{D}^{e t}\left(\varphi_{\Delta}, \Gamma_{\Delta}, E_{\Delta}\right)$ with $\operatorname{Rep}_{\mathbb{F}_{p}}\left(G_{\mathbb{Q}_{p}, \Delta}\right)$ we shall see later on (Cor. 3.16) that any object $D$ in $\mathcal{D}^{e t}\left(\varphi_{\Delta}, \Gamma_{\Delta}, E_{\Delta}\right)$ is in fact free over $E_{\Delta}$.

We equip $E_{\Delta}^{+}$with the $X_{\Delta}$-adic topology. Then $\left(E_{\Delta}, E_{\Delta}^{+}\right)$is a Huber pair (in the sense of [7]) if we equip $E_{\Delta}$ with the inductive limit topology $E_{\Delta}=\bigcup_{n} X_{\Delta}^{-n} E_{\Delta}^{+}$. In fact, $E_{\Delta}$ is a complete noetherian Tate ring (op. cit.). Note that this is not the natural compact topology on $E_{\Delta}^{+}$as in the compact topology $E_{\Delta}^{+}$would not be open in $E_{\Delta}$ since the index of $E_{\Delta}^{+}$in $X_{\Delta}^{-n} E_{\Delta}^{+}$is not finite. On the other hand, the inclusion $\mathbb{F}_{p}\left(\left(X_{\alpha}\right)\right) \hookrightarrow E_{\Delta}$ is not continuous in the $X_{\Delta}$-adic topology therefore we cannot apply Drinfeld's Lemma (Thm. 17.2.4 in [7]) directly in this situation.

Let $D$ be an object in $\mathcal{D}^{e t}\left(\varphi_{\Delta}, \Gamma_{\Delta}, E_{\Delta}\right)$. By Banach's Theorem for Tate rings (Prop. 6.18 in [8]), there is a unique $E_{\Delta}$-module topology on $D$ that we call the $X_{\Delta}$-adic topology. Moreover, any $E_{\Delta}$-module homomorphism is continuous in the $X_{\Delta}$-adic topology.

\section{$2.2 \quad$ Integrality properties}

Put $\varphi_{s}:=\prod_{\alpha \in \Delta} \varphi_{\alpha} \in T_{+, \Delta}$ and define $D^{++}:=\left\{x \in D \mid \lim _{k \rightarrow \infty} \varphi_{s}^{k}(x)=0\right\}$ where the limit is considered in the $X_{\Delta}$-adic topology (cf. II.2.1 in [3] in case $|\Delta|=1$ ). Note that $\varphi_{s}$ is the absolute Frobenius on $E_{\Delta}$, it takes any element to its $p$ th power.

Lemma 2.4. Let $M$ be a finitely generated $E_{\Delta}^{+}$-submodule in $D$. Then $E_{\Delta}^{+} \varphi_{s}(M)$ is also finitely generated.

Proof. If $M$ is generated by $m_{1}, \ldots, m_{n}$ then $\varphi_{s}\left(m_{1}\right), \ldots, \varphi_{s}\left(m_{n}\right)$ generate $E_{\Delta}^{+} \varphi_{s}(M)$.

Proposition 2.5. $D^{++}$is a finitely generated $E_{\Delta}^{+}$-submodule in $D$ that is stable under the action of $T_{+, \Delta}$ and we have $D=D^{++}\left[X_{\Delta}^{-1}\right]$.

Proof. Choose an arbitrary finitely generated $E_{\Delta}^{+}$-submodule $M$ of $D$ with $M\left[X_{\Delta}^{-1}\right]=D$ (e.g. take $M=E_{\Delta}^{+} e_{1}+\cdots+E_{\Delta}^{+} e_{n}$ for some $E_{\Delta}$-generating system $e_{1}, \ldots, e_{n}$ of $\left.D\right)$. By Lemma 2.4 we have an integer $r \geq 0$ such that $\varphi(M) \subseteq X_{\Delta}^{-r} M$, since $E_{\Delta}^{+}$is noetherian and we have $D=\bigcup_{r} X_{\Delta}^{-r} M$. Then we have

$$
\varphi_{s}\left(X_{\Delta}^{k} M\right)=X_{\Delta}^{p k} \varphi_{s}(M) \subseteq X_{\Delta}^{p k-r} M \subseteq X_{\Delta}^{k+1} M
$$

for any integer $k \geq \frac{r+1}{p-1}$. Therefore we have $X_{\Delta}^{\left[\frac{r+1}{p-1}\right]+1} M \subseteq D^{++}$whence $D^{++}\left[X_{\Delta}^{-1}\right]=$ $M\left[X_{\Delta}^{-1}\right]=D$. 
Since $T_{+\Delta}$ is commutative and the action of each $\varphi_{t}\left(t \in T_{+, \Delta}\right)$ is continuous, $D^{++}$is stable under the action of $T_{+, \Delta}$. There is a system of neighbourhoods of 0 in $D$ consisting of $E_{\Delta}^{+}$-submodules therefore $D^{++}$is an $E_{\Delta}^{+}$-submodule.

To prove that $D^{++}$is finitely generated over $E_{\Delta}^{+}$suppose first that $D$ is a free module over $E_{\Delta}$ generated by $e_{1}, \ldots, e_{n}$ and put $M:=E_{\Delta}^{+} e_{1}+\cdots+E_{\Delta}^{+} e_{n}$. We may assume $M \subseteq D^{++}$by replacing $M$ with $X_{\Delta}^{\left[\frac{r+1}{p-1}\right]+1} M$. Moreover, further multiplying $M=E_{\Delta}^{+} e_{1}+\cdots+E_{\Delta}^{+} e_{n}$ by a power of $X_{\Delta}$, we may assume that the matrix $A:=\left[\varphi_{s}\right]_{e_{1}, \ldots, e_{n}}$ of $\varphi_{s}$ in the basis $e_{1}, \ldots, e_{n}$ lies in $E_{\Delta}^{+n \times n}$ as we have $\left[\varphi_{s}\right]_{X_{\Delta}^{r} e_{1}, \ldots, X_{\Delta}^{r} e_{n}}=X_{\Delta}^{(p-1) r}\left[\varphi_{s}\right]_{e_{1}, \ldots, e_{n}}$. Now we choose the integer $r>0$ so that it is bigger than $\operatorname{val}_{X_{\alpha}}(\operatorname{det} A)$ for all $\alpha \in \Delta$ and claim that $D^{++} \subseteq X_{\Delta}^{-r} M$ whence $D^{++}$is finitely generated over $E_{\Delta}^{+}$as $E_{\Delta}^{+}$is noetherian. Assume for contradiction that $d=\sum_{i=1}^{n} d_{i} e_{i}$ lies in $D^{++}$for some $d_{i} \in E_{\Delta}(i=1, \ldots, n)$ such that at least one $d_{i}$, say $d_{1}$, does not lie in $X_{\Delta}^{-r} E_{\Delta}^{+}$. In particular, there exists an $\alpha$ in $\Delta$ such that $\operatorname{val}_{X_{\alpha}}\left(d_{1}\right)<-r$. Since $M$ is open in $D$ and $d \in D^{++}$, there exists an integer $k>0$ such that $\varphi_{s}^{k}(d)$ is in $M$ which is equivalent to saying that the column vector

$$
A \varphi_{s}(A) \ldots \varphi_{s}^{k-1}(A)\left(\begin{array}{c}
\varphi_{s}^{k}\left(d_{1}\right) \\
\vdots \\
\varphi_{s}^{k}\left(d_{n}\right)
\end{array}\right)
$$

lies in $E_{\Delta}^{+n}$. Multiplying this by the matrix built from the $(n-1) \times(n-1)$ minors of $A \varphi_{s}(A) \ldots \varphi_{s}^{k-1}(A)$ we $\operatorname{deduce}$ that $\operatorname{det}\left(A \varphi_{s}(A) \ldots \varphi_{s}^{k-1}(A)\right) \varphi_{s}^{k}\left(d_{1}\right)=\operatorname{det}(A)^{\frac{p^{k}-1}{p-1}} d_{1}^{p^{k}}$ lies in $E_{\Delta}^{+}$. We compute

$$
\begin{array}{r}
0 \leq \operatorname{val}_{X_{\alpha}}\left(\operatorname{det}(A)^{\frac{p^{k}-1}{p-1}} d_{1}^{p^{k}}\right)=\frac{p^{k}-1}{p-1} \operatorname{val}_{X_{\alpha}}(\operatorname{det}(A))+p^{k} \operatorname{val}_{X_{\alpha}}\left(d_{1}\right)< \\
<\frac{p^{k}-1}{p-1} \operatorname{val}_{X_{\alpha}}(\operatorname{det}(A))-p^{k} r<0
\end{array}
$$

by our assumption that $r>\operatorname{val}_{X_{\alpha}}(\operatorname{det}(A))$, yielding a contradiction.

In the general case note that $D$ is always stably free by Prop. 2.2 and Lemma 2.3. So $D_{1}:=D \oplus E_{\Delta}^{k}$ is a free module over $E_{\Delta}$ for $k$ large enough. We make $D_{1}$ into an étale $T_{+, \Delta}$-module by the trivial action of $T_{+, \Delta}$ on $E_{\Delta}^{k}$ to deduce that $D_{1}^{++}$is finitely generated over $E_{\Delta}^{+}$. The result follows noting that $D^{++} \subseteq D_{1}^{++}$and $E_{\Delta}^{+}$is noetherian.

For an object $D$ in $\mathcal{D}^{e t}\left(\varphi_{\Delta}, \Gamma_{\Delta}, E_{\Delta}\right)$ we define

$$
D^{+}:=\left\{x \in D \mid\left\{\varphi_{s}^{k}(x): k \geq 0\right\} \subset D \text { is bounded }\right\}
$$

Since $\varphi_{s}^{k}\left(X_{\Delta}\right)$ tends to 0 in the $X_{\Delta^{-}}$adic topology, we have $X_{\Delta} D^{+} \subseteq D^{++}$, ie. $D^{+} \subseteq X_{\Delta}^{-1} D^{++}$. In particular, $D^{+}$is finitely generated over $E_{\Delta}^{+}$. On the other hand, we also have $D^{++} \subseteq D^{+}$ by construction whence we deduce $D=D^{+}\left[X_{\Delta}^{-1}\right]$.

Lemma 2.6. We have $\varphi_{t}\left(D^{+}\right) \subset D^{+}\left(\right.$resp. $\left.\varphi_{t}\left(D^{++}\right) \subset D^{++}\right)$for all $\varphi_{t} \in T_{+, \Delta}$.

Proof. For any generating system $e_{1}, \ldots, e_{n}$ of $D$ and any $\varphi_{t} \in T_{+, \Delta}$ there exists an integer $k=k\left(\varphi_{t}, M\right)>0$ such that we have $\varphi_{t}\left(X_{\Delta}^{k} M\right) \subseteq X_{\Delta}^{k} E_{\Delta}^{+} \varphi_{t}(M) \subseteq M$ where we put $M:=$ 
$E_{\Delta}^{+} e_{1}+\cdots+E_{\Delta}^{+} e_{n}$ by Lemma 2.4 . Indeed, $X_{\Delta}$ divides $\varphi_{t}\left(X_{\Delta}\right)$ in $E_{\Delta}^{+}$, and we have $D=$ $M\left[1 / X_{\Delta}\right]$ by construction. The statement on $D^{++}$follows from the commutativity of the monoid $T_{+, \Delta}$ noting that there exists a basis of neighbouhoods of 0 in $D$ consisting of $E_{\Delta^{-}}^{+}$ submodules of the form $M$. To see that $\varphi_{t}\left(D^{+}\right) \subseteq D^{+}$note that $\varphi_{t}\left(D^{+}\right)$is bounded and we have $\varphi_{s}^{k}\left(\varphi_{t}\left(D^{+}\right)\right)=\varphi_{t}\left(\varphi_{s}^{k}\left(D^{+}\right)\right) \subset \varphi_{t}\left(D^{+}\right)$.

Now fix an $\alpha \in \Delta$ and define $D_{\bar{\alpha}}^{+}:=D^{+}\left[X_{\Delta \backslash\{\alpha\}}^{-1}\right]$ where for any subset $S \subseteq \Delta$ we put $X_{S}:=\prod_{\beta \in S} X_{\beta}$. Then $D_{\bar{\alpha}}^{+}$is a finitely generated module over $E_{\bar{\alpha}}^{+}:=E_{\Delta}^{+}\left[X_{\Delta \backslash\{\alpha\}}^{-1}\right]$. We denote by $T_{+, \bar{\alpha}} \subset T_{+, \Delta}$ the monoid generated by $\varphi_{\beta}(\beta \in \Delta \backslash\{\alpha\})$ and $\Gamma_{\Delta}$.

Lemma 2.7. $D_{\bar{\alpha}}^{+} / D^{+}$is $X_{\alpha}$-torsion free: If both $X_{\alpha}^{n_{1}} d$ and $X_{\Delta \backslash\{\alpha\}}^{n_{2}} d$ lie in $D^{+}$for some element $d \in D^{+}, \alpha \in \Delta$, and integers $n_{1}, n_{2} \geq 0$ then we have $d \in D^{+}$. The same statement holds if we replace $D^{+}$by $D^{++}$.

Proof. At first assume that $D$ is free as a module over $E_{\Delta}$ with basis $e_{1}, \ldots, e_{n}$. Then the denominators of $\varphi_{s}^{k}\left(X_{\alpha}^{n_{1}} d\right)=X_{\alpha}^{n_{1} p^{k}} \varphi_{s}^{k}(d)$ in the basis $e_{1}, \ldots, e_{n}$ are bounded for $k \geq 0$ by assumption. Therefore the $X_{\beta}$-valuations of the denominators of $\varphi_{s}^{k}(d)$ are bounded for all $\beta \in \Delta \backslash\{\alpha\}$ since $E_{\Delta}^{+}$is a unique factorization domain. On the other hand, the $X_{\alpha}$-valuations of these denominators are also bounded since the denominators of $\varphi_{s}^{k}\left(X_{\Delta \backslash\{\alpha\}}^{n_{2}} d\right)=X_{\Delta \backslash\{\alpha\}}^{n_{2} p^{k}} \varphi_{s}^{k}(d)$ are bounded. To prove the statement we have the same argument but 'being bounded' replaced by 'tends to 0 '.

Finally, by Prop. 2.2 and Lemma $2.3 D \oplus E_{\Delta}^{k}$ is free over $E_{\Delta}$ and we equip it with the structure of an étale $(\varphi, \Gamma)$-module (trivially on $E_{\Delta}^{k}$ ). The statement follows from the additivity of the constructions $D \mapsto D^{+}$and $D \mapsto D_{\bar{\alpha}}^{+}$in direct sums.

Lemma 2.8. Assume that $D$ is generated by a single element $e_{1} \in D$ over $E_{\Delta}$. Then for any $\varphi_{t}$ in $T_{+, \bar{\alpha}}$ we have $\varphi_{t}\left(e_{1}\right)=a_{t} e_{1}$ for some unit $a_{t}$ in $\left(E_{\bar{\alpha}}^{+}\right)^{\times}$.

Proof. Define $a_{t} \in E_{\Delta}$ and $a_{\alpha} \in E_{\Delta}$ so that $\varphi_{t}\left(e_{1}\right)=a_{t} e_{1}$ and $\varphi_{\alpha}\left(e_{1}\right)=a_{\alpha} e_{1}$. By the étale property both $a_{t}$ and $a_{\alpha}$ are units in $E_{\Delta}$, so it remains to show that $\operatorname{val}_{X_{\alpha}}\left(a_{t}\right)=0$. We compute

$$
\begin{aligned}
& \varphi_{\alpha}\left(a_{t}\right) a_{\alpha} e_{1}=\varphi_{\alpha}\left(a_{t}\right) \varphi_{\alpha}\left(e_{1}\right)=\varphi_{\alpha}\left(a_{t} e_{1}\right)=\varphi_{\alpha}\left(\varphi_{t}\left(e_{1}\right)\right)= \\
& =\varphi_{t}\left(\varphi_{\alpha}\left(e_{1}\right)\right)=\varphi_{t}\left(a_{\alpha} e_{1}\right)=\varphi_{t}\left(a_{\alpha}\right) \varphi_{t}\left(e_{1}\right)=\varphi_{t}\left(a_{\alpha}\right) a_{t} e_{1}
\end{aligned}
$$

whence we deduce

$$
p \operatorname{val}_{X_{\alpha}}\left(a_{t}\right)+\operatorname{val}_{X_{\alpha}}\left(a_{\alpha}\right)=\operatorname{val}_{X_{\alpha}}\left(\varphi_{\alpha}\left(a_{t}\right) a_{\alpha}\right)=\operatorname{val}_{X_{\alpha}}\left(\varphi_{t}\left(a_{\alpha}\right) a_{t}\right)=\operatorname{val}_{X_{\alpha}}\left(a_{\alpha}\right)+\operatorname{val}_{X_{\alpha}}\left(a_{t}\right) .
$$

This yields $\operatorname{val}_{X_{\alpha}}\left(a_{t}\right)=0$ as required.

Lemma 2.9. There exists an integer $k=k(D)>0$ such that for any $\varphi_{t} \in T_{+, \bar{\alpha}}$ we have $X_{\alpha}^{k} D_{\bar{\alpha}}^{+} \subseteq E_{\Delta}^{+} \varphi_{t}\left(D_{\bar{\alpha}}^{+}\right)$.

Proof. At first assume that $D$ is free, choose a basis $e_{1}, \ldots, e_{n}$ contained in $D^{+}$, and put $M:=E_{\Delta}^{+} e_{1}+\ldots E_{\Delta}^{+} e_{n}, M_{\alpha}:=E_{\bar{\alpha}}^{+} e_{1}+\cdots+E_{\bar{\alpha}}^{+} e_{n}$. There exists an integer $k_{0}>0$ such that $D^{+} \subseteq X_{\Delta}^{-k_{0}} M$. In particular, we have $D_{\bar{\alpha}}^{+} \subseteq X_{\alpha}^{-k_{0}} M_{\bar{\alpha}}$. Now for a fixed $\varphi_{t} \in T_{+, \bar{\alpha}}$ let $A_{t} \in E_{\Delta}^{n \times n}$ be the matrix of $\varphi_{t}$ in the basis $e_{1}, \ldots, e_{n}$. Since $\varphi_{t}\left(e_{i}\right)$ lies in $D^{+} \subseteq X_{\alpha}^{-k_{0}} M_{\bar{\alpha}}$, 
all the entries of the matrix $A_{t}$ are in $X_{\alpha}^{-k_{0}} E_{\bar{\alpha}}^{+}$. Applying Lemma 2.8 to the single generator $e_{1} \wedge \cdots \wedge e_{n}$ of $\bigwedge^{n} D$ we obtain $\operatorname{val}_{X_{\alpha}}\left(\operatorname{det} A_{t}\right)=0$. In particular, all the entries of $A_{t}^{-1}$ lie in $X_{\alpha}^{-(n-1) k_{0}} E_{\bar{\alpha}}^{+}$by the formula for the inverse matrix using the $(n-1) \times(n-1)$ minors in $A_{t}$. Now note that the elements $e_{1}, \ldots, e_{n}$ can be written as a linear combination of $\varphi_{t}\left(e_{1}\right), \ldots, \varphi_{t}\left(e_{n}\right)$ with coefficients from $A_{t}^{-1}$. Using Lemma 2.6 this shows

$$
X_{\alpha}^{k_{0}} D_{\bar{\alpha}}^{+} \subseteq M_{\bar{\alpha}} \subseteq X_{\alpha}^{-(n-1) k_{0}} \varphi_{t}\left(M_{\bar{\alpha}}\right) \subseteq X_{\alpha}^{-(n-1) k_{0}} D_{\bar{\alpha}}^{+}
$$

So we may choose $k:=n k_{0}$ independent of $\varphi_{t}$.

The general case follows from Prop. 2.2 and Lemma 2.3 noting that the functor $D \mapsto D_{\bar{\alpha}}^{+}$ commutes with direct sums.

In view of the above Lemma we define

$$
D_{\bar{\alpha}}^{+*}:=\bigcap_{\varphi t \in T_{+, \bar{\alpha}}} E_{\bar{\alpha}}^{+} \varphi_{t}\left(D_{\bar{\alpha}}^{+}\right)
$$

$D_{\bar{\alpha}}^{+*}$ is finitely generated over $E_{\bar{\alpha}}^{+}$as it is contained in $D_{\bar{\alpha}}^{+}$and $E_{\bar{\alpha}}^{+}$is noetherian. On the other hand, by Lemma 2.9 we have $X_{\alpha}^{k} D_{\bar{\alpha}}^{+} \subseteq D_{\bar{\alpha}}^{+*}$ for some integer $k=k(D)>0$ whence, in particular, $D=D_{\bar{\alpha}}^{+*}\left[X_{\alpha}^{-1}\right]$.

Proposition 2.10. $D_{\bar{\alpha}}^{+*}$ is an étale $T_{+, \bar{\alpha}}$ module over $E_{\bar{\alpha}}^{+}$, ie. the maps

$$
\operatorname{id} \otimes \varphi_{t}: \varphi_{t}^{*} D_{\bar{\alpha}}^{+*}=E_{\bar{\alpha}}^{+} \otimes_{E_{\bar{\alpha}}^{+}, \varphi_{t}} D_{\bar{\alpha}}^{+*} \rightarrow D_{\bar{\alpha}}^{+*}
$$

are bijective for all $\varphi_{t} \in T_{+, \alpha}$.

Proof. At first note that we have $\varphi_{t}\left(D_{\bar{\alpha}}^{+*}\right) \subseteq D_{\bar{\alpha}}^{+*}$ for all $\varphi_{t} \in T_{+, \bar{\alpha}}$ by Lemma 2.6 and the commutativity of $T_{+, \bar{\alpha}}$, so the map (2) exists. Now let $\varphi_{t_{0}} \in T_{+, \bar{\alpha}}$ be arbitrary. Since $E_{\bar{\alpha}}^{+}$(resp. $\left.E_{\Delta}\right)$ is a finite free module over $\varphi_{t_{0}}\left(E_{\bar{\alpha}}^{+}\right)$(resp. over $\varphi_{t_{0}}\left(E_{\Delta}\right)$ ) with generators contained in $E_{\Delta}^{+}$, we have a natural identification $\varphi_{t_{0}}^{*} D_{\bar{\alpha}}^{+*} \cong E_{\Delta}^{+} \otimes_{E_{\Delta}^{+}, \varphi_{t_{0}}} D_{\Delta}^{+*}\left(\operatorname{resp} . \varphi_{t_{0}}^{*} D \cong E_{\Delta}^{+} \otimes_{E_{\Delta}^{+}, \varphi_{0}} D\right)$. Since $E_{\Delta}^{+}$is finite free (hence flat) over $\varphi_{t_{0}}\left(E_{\Delta}^{+}\right)$, the inclusion $D_{\bar{\alpha}}^{+} \subset D$ induces an inclusion $\varphi_{t_{0}}^{*} D_{\bar{\alpha}}^{+} \subset \varphi_{t_{0}}^{*} D$. It follows that (2) is injective since $D$ is étale. Similarly, for each $\varphi_{t} \in T_{+, \bar{\alpha}}$, the map

$$
\mathrm{id} \otimes \varphi_{t_{0}}: \varphi_{t_{0}}^{*}\left(E_{\bar{\alpha}}^{+} \varphi_{t}\left(D_{\bar{\alpha}}^{+}\right)\right) \rightarrow E_{\bar{\alpha}}^{+} \varphi_{t}\left(D_{\bar{\alpha}}^{+}\right)
$$

is injective with image $E_{\bar{\alpha}}^{+} \varphi_{t_{0}} \varphi_{t}\left(D_{\bar{\alpha}}^{+}\right)$. On the other hand, since $E_{\Delta}^{+}$is finite free over $\varphi_{t_{0}}\left(E_{\Delta}^{+}\right)$, we have $\varphi_{t_{0}}^{*} D_{\bar{\alpha}}^{+*}=\bigcap_{t \in T_{+, \bar{\alpha}}} \varphi_{t_{0}}^{*}\left(E_{\bar{\alpha}}^{+} \varphi_{t}\left(D_{\bar{\alpha}}^{+}\right)\right)$where the intersection is taken inside $\varphi_{t_{0}}^{*} D$. Therefore (2) is bijective as we have $D_{\bar{\alpha}}^{+*}=\bigcap_{\varphi_{t} \in T_{+, \bar{\alpha}}} E_{\bar{\alpha}}^{+} \varphi_{t_{0}} \varphi_{t}\left(D_{\bar{\alpha}}^{+}\right)$.

Lemma 2.11. There exists a finitely generated $E_{\Delta}^{+}$-submodule $D_{0} \subset D_{\bar{\alpha}}^{+*}$ such that $D_{0} \subseteq$ $E_{\Delta}^{+} \varphi_{\bar{\alpha}}\left(D_{0}\right)$ and $D_{\bar{\alpha}}^{+*}=D_{0}\left[X_{\Delta \backslash\{\alpha\}}^{-1}\right]$ where $\varphi_{\bar{\alpha}}:=\prod_{\beta \in \Delta \backslash\{\alpha\}} \varphi_{\beta}$. Moreover, we have $D_{\bar{\alpha}}^{+*}=$ $\bigcup_{r \geq 0} E_{\Delta}^{+} \varphi_{\bar{\alpha}}^{r}\left(X_{\Delta \backslash\{\alpha\}}^{-1} D_{0}\right)$.

Proof. Put $D_{1}:=D^{+} \cap D_{\bar{\alpha}}^{+*}$. By Prop. 2.10 and the fact that $D_{\bar{\alpha}}^{+*}=D_{1}\left[X_{\Delta \backslash\{\alpha\}}^{-1}\right]$ we find an integer $k_{0}>0$ such that $X_{\Delta \backslash\{\alpha\}}^{k_{0}} D_{1} \subseteq E_{\Delta}^{+} \varphi_{\bar{\alpha}}\left(D_{1}\right)$. So for $k>\frac{k_{0}}{p-1}$ we have

$$
X_{\Delta \backslash\{\alpha\}}^{-k} D_{1} \subseteq X_{\Delta \backslash\{\alpha\}}^{-k-k_{0}} E_{\Delta}^{+} \varphi_{\bar{\alpha}}\left(D_{1}\right) \subseteq X_{\Delta \backslash\{\alpha\}}^{-p k} E_{\Delta}^{+} \varphi_{\bar{\alpha}}\left(D_{1}\right)=E_{\Delta}^{+} \varphi_{\bar{\alpha}}\left(X_{\Delta \backslash\{\alpha\}}^{-k} D_{1}\right) .
$$


So we put $D_{0}:=X_{\Delta \backslash\{\alpha\}}^{-k} D_{1}$ so that the first part of the statement is satisfied. Iterating the inclusion $D_{0} \subseteq E_{\Delta}^{+} \varphi_{\bar{\alpha}}\left(D_{0}\right)$ we obtain $D_{0} \subseteq E_{\Delta}^{+} \varphi_{\bar{\alpha}}^{r}\left(D_{0}\right)$ for all $r \geq 1$. Finally, we compute

$$
X_{\Delta \backslash\{\alpha\}}^{-p^{r}} D_{0} \subseteq X_{\Delta \backslash\{\alpha\}}^{-p^{r}} E_{\Delta}^{+} \varphi_{\bar{\alpha}}^{r}\left(D_{0}\right)=E_{\Delta}^{+} \varphi_{\bar{\alpha}}^{r}\left(X_{\Delta \backslash\{\alpha\}}^{-1} D_{0}\right)
$$

The statement follows noting that we have $D_{\bar{\alpha}}^{+*}=D_{0}\left[X_{\Delta \backslash\{\alpha\}}^{-1}\right]=\bigcup_{r} X_{\Delta \backslash\{\alpha\}}^{-p^{r}} D_{0}$.

\section{The equivalence of categories for $\mathbb{F}_{p}$-representations}

\subsection{The functor $\mathbb{D}$}

Take a copy $G_{\mathbb{Q}_{p}, \alpha} \cong \operatorname{Gal}\left(\overline{\mathbb{Q}_{p}} / \mathbb{Q}_{p}\right)$ of the absolute Galois group of $\mathbb{Q}_{p}$ for each element $\alpha \in \Delta$ and let $G_{\mathbb{Q}_{p}, \Delta}:=\prod_{\alpha \in \Delta} G_{\mathbb{Q}_{p}, \alpha}$. Let $\operatorname{Rep}_{\mathbb{F}_{p}}\left(G_{\mathbb{Q}_{p}, \Delta}\right)$ be the category of continuous representations of the group $G_{\mathbb{Q}_{p}, \Delta}$ on finite dimensional $\mathbb{F}_{p}$ vectorspaces. We identify $\Gamma_{\alpha}$ with the Galois group $\operatorname{Gal}\left(\mathbb{Q}_{p}\left(\mu_{p^{\infty}}\right) / \mathbb{Q}_{p}\right)$ as a quotient of $G_{\mathbb{Q}_{p}, \alpha}$ via the cyclotomic character $\chi_{\alpha}: \operatorname{Gal}\left(\mathbb{Q}_{p}\left(\mu_{p^{\infty}}\right) / \mathbb{Q}_{p}\right) \rightarrow \mathbb{Z}_{p}^{\times}$. Further, we denote by $H_{\mathbb{Q}_{p}, \alpha}$ the kernel of the natural quotient map $G_{\mathbb{Q}_{p}, \alpha} \rightarrow \Gamma_{\alpha}$ and put $H_{\mathbb{Q}_{p}, \Delta}:=\prod_{\alpha \in \Delta} H_{\mathbb{Q}_{p}, \alpha} \triangleleft G_{\mathbb{Q}_{p}, \Delta}$. Putting $E_{\alpha}:=\mathbb{F}_{p}\left(\left(X_{\alpha}\right)\right)$ we have the following fundamental result of Fontaine and Wintenberger (Thm. 4.16 [5]).

Theorem 3.1. The absolute Galois group $\operatorname{Gal}\left(E_{\alpha}^{s e p} / E_{\alpha}\right)$ is isomorphic to $H_{\mathbb{Q}_{p}, \alpha}$. Moreover, $G_{\mathbb{Q}_{p}, \alpha}$ acts on the separable closure $E_{\alpha}^{\text {sep }}$ via automorphisms such that the action of $\Gamma_{\alpha} \cong$ $G_{\mathbb{Q}_{p}, \alpha} / H_{\mathbb{Q}_{p}, \alpha}$ on $E_{\alpha}=\left(E_{\alpha}^{s e p}\right)^{H_{\mathbb{Q}_{p}, \alpha}}$ coincides with the one given in (1).

For each $\alpha \in \Delta$ consider a finite separable extension $E_{\alpha}^{\prime}$ of $E_{\alpha}$ together with the Frobenius $\varphi_{\alpha}: E_{\alpha}^{\prime} \rightarrow E_{\alpha}^{\prime}$ acting by raising to the power $p$. We denote by $E_{\alpha}^{\prime+}$ the integral closure of $E_{\alpha}^{+}=\mathbb{F}_{p}\left[\left[X_{\alpha}\right]\right]$ in $E_{\alpha}^{\prime}$. Note that $E_{\alpha}^{\prime}$ is isomorphic to $\mathbb{F}_{q_{\alpha}}\left(\left(X_{\alpha}^{\prime}\right)\right)$ for some power $q_{\alpha}$ of $p$ and uniformizer $X_{\alpha}^{\prime}$ such that we have $E_{\alpha}^{\prime+} \cong \mathbb{F}_{q_{\alpha}}\left[\left[X_{\alpha}^{\prime}\right]\right]$. We normalize the $X_{\alpha}$-adic (multiplicative) valuation on $E_{\alpha}$ so that we have $\left|X_{\alpha}\right|_{X_{\alpha}}=p^{-1}$. This extends uniquely to the finite extension $E_{\alpha}^{\prime}$. Moreover, we equip the tensor product $E_{\Delta, \circ}^{\prime}:=\bigotimes_{\alpha \in \Delta, \mathbb{F}_{p}} E_{\alpha}^{\prime}$ with a norm $|\cdot|_{\text {prod }}$ by the formula

$$
|c|_{\text {prod }}:=\inf \left(\max _{i}\left(\prod_{\alpha \in \Delta}\left|c_{\alpha, i}\right|_{\alpha}\right) \mid c=\sum_{i=1}^{n} \bigotimes_{\alpha \in \Delta} c_{\alpha, i}\right) .
$$

Note that the restriction of $|\cdot|_{\text {prod }}$ to the subring $E_{\Delta, \circ}^{\prime+}:=\bigotimes_{\alpha \in \Delta, \mathbb{F}_{p}} E_{\alpha}^{\prime+}$ induces the valuation with respect to the augmentation ideal $\operatorname{Ker}\left(E_{\Delta, \circ}^{\prime+} \rightarrow \bigotimes_{\alpha \in \Delta, \mathbb{F}_{p}} \mathbb{F}_{q_{\alpha}}\right)$. The norm $|\cdot|_{\text {prod }}$ is not multiplicative in general, as the ring $\left.\bigotimes_{\alpha \in \Delta, \mathbb{F}_{p}} \mathbb{F}_{q_{\alpha}}\right)$ is not a domain. However, it is submultiplicative. We define $E_{\Delta}^{\prime+}$ as the completion of $E_{\Delta, \mathrm{o}}^{\prime+}$ with respect to $|\cdot|_{\text {prod }}$ and put $E_{\Delta}^{\prime}:=E_{\Delta}^{\prime+}\left[1 / X_{\Delta}\right]$. Note that $E_{\Delta}^{\prime}$ is not complete with respect to $|\cdot|_{\text {prod }}$ (unless $|\Delta|=1$ ) even though $E_{\Delta, \circ}^{\prime}=E_{\Delta, o}^{\prime+}\left[1 / X_{\Delta}\right]$ is a dense subring in $E_{\Delta}^{\prime}$. Since we have a containment

$$
\left(\bigotimes_{\alpha \in \Delta, \mathbb{F}_{p}} \mathbb{F}_{q_{\alpha}}\right)\left[X_{\alpha}^{\prime}, \alpha \in \Delta\right]=\bigotimes_{\alpha \in \Delta, \mathbb{F}_{p}} \mathbb{F}_{q_{\alpha}}\left[X_{\alpha}\right] \leq_{\text {dense }} E_{\Delta, \circ}^{\prime+}
$$

we may identify $E_{\Delta}^{\prime+}$ with the power series $\operatorname{ring}\left(\bigotimes_{\alpha \in \Delta, \mathbb{F}_{p}} \mathbb{F}_{q_{\alpha}}\right)\left[\left[X_{\alpha}^{\prime}, \alpha \in \Delta\right]\right]$ which is the completion of the polynomial ring above. In particular, the special case $E_{\alpha}^{\prime}=E_{\alpha}$ for all $\alpha \in \Delta$ yields a ring $E_{\Delta}^{\prime}$ isomorphic to $E_{\Delta}$. Therefore $E_{\Delta}$ is a subring of $E_{\Delta}^{\prime}$ for all collection 
of finite separable extensions $E_{\alpha}^{\prime}$ of $E_{\alpha}(\alpha \in \Delta)$. Further, $\varphi_{\alpha}$ acts on $E_{\Delta, \circ}^{\prime+}$ (and on $E_{\Delta, \circ}^{\prime}$ ) by the Frobenius on the component in $E_{\alpha}^{\prime}$ and by the identity on all the other components in $E_{\beta}^{\prime}$, $\beta \in \Delta \backslash\{\alpha\}$. This action is continuous in the norm $|\cdot|_{\text {prod }}$ therefore extends to the completion $E_{\Delta}^{\prime+}$ and the localization $E_{\Delta}^{\prime}$. We have the following alternative characterization of the ring $E_{\Delta}^{\prime}$.

Lemma 3.2. Put $\Delta=\left\{\alpha_{1}, \ldots, \alpha_{n}\right\}$. We have

$$
E_{\Delta}^{\prime} \cong E_{\alpha_{1}}^{\prime} \otimes_{E_{\alpha_{1}}}\left(E_{\alpha_{2}}^{\prime} \otimes_{E_{\alpha_{2}}}\left(\cdots\left(E_{\alpha_{n}}^{\prime} \otimes_{E_{\alpha_{n}}} E_{\Delta}\right)\right)\right)
$$

Proof. By rearranging the order of tensor products we have an identification

$$
E_{\Delta, \circ}^{\prime+}=\bigotimes_{\alpha \in \Delta, \mathbb{F}_{p}}\left(E_{\alpha}^{\prime+} \otimes_{E_{\alpha}^{+}} E_{\alpha}^{+}\right) \cong E_{\alpha_{1}}^{\prime+} \otimes_{E_{\alpha_{1}}^{+}}\left(E_{\alpha_{2}}^{\prime+} \otimes_{E_{\alpha_{2}}^{+}}\left(\ldots\left(E_{\alpha_{n}}^{\prime+} \otimes_{E_{\alpha_{n}}^{+}} E_{\Delta, o}^{+}\right)\right)\right)
$$

The statement follows by completing this with respect to the maximal ideal of $E_{\Delta}^{+}$and inverting $X_{\Delta}$.

We define the multivariable analogue of $E^{\text {sep }}$ as

$$
E_{\Delta}^{s e p}:=\underset{E_{\alpha} \leq E_{\alpha}^{\prime} \leq E_{\alpha}^{s e p}, \forall \alpha \in \Delta}{\lim _{\Delta}^{\prime}} E_{\Delta}^{\prime}
$$

For any subset $S \subseteq \Delta$ we define the similar notions $E_{S}^{\prime+}, E_{S}^{\prime}$, and $E_{S}^{s e p}$ with $\Delta$ replaced by $S$. We equip $E_{\Delta}^{s e p}$ with the relative Frobenii $\varphi_{\alpha}$ for each $\alpha \in \Delta$ defined above on each $E_{\Delta}^{\prime}$. Further, $E_{\Delta}^{s e p}$ admits an action of $G_{\mathbb{Q}_{p}, \Delta}$ satisfying

Proposition 3.3. Assume that the extensions $E_{\alpha}^{\prime} / E_{\alpha}$ are Galois for all $\alpha \in \Delta$ and let $H^{\prime}:=\prod_{\alpha \in \Delta} H_{\alpha}^{\prime}$ where $H_{\alpha}^{\prime}:=\operatorname{Gal}\left(E_{\alpha}^{\text {sep }} / E_{\alpha}^{\prime}\right)$. Then we have $\left(E_{\Delta}^{\text {sep }}\right)^{H_{\Delta}^{\prime}}=E_{\Delta}^{\prime}$. In particular, the subring $\left(E_{\Delta}^{s e p}\right)^{H_{\mathbb{Q}_{p}, \Delta}}$ of $H_{\mathbb{Q}_{p}, \Delta}$-invariants in $E_{\Delta}^{\text {sep }}$ equals $E_{\Delta}$ with the previously defined action of $\Gamma_{\Delta} \cong G_{\mathbb{Q}_{p}, \Delta} / H_{\mathbb{Q}_{p}, \Delta}$.

Proof. Since $X_{\Delta}$ is $H_{\Delta}^{\prime}$-invariant and $\lim _{\longrightarrow}$ can be interchanged with taking $H_{\Delta}^{\prime}$-invariants, it suffices to show that whenever

$$
E_{\alpha}=\mathbb{F}_{p}\left(\left(X_{\alpha}\right)\right) \leq E_{\alpha}^{\prime}=\mathbb{F}_{q_{\alpha}^{\prime}}\left(\left(X_{\alpha}^{\prime}\right)\right) \leq E_{\alpha}^{\prime \prime}=\mathbb{F}_{q_{\alpha}^{\prime \prime}}\left(\left(X_{\alpha}^{\prime \prime}\right)\right)
$$

is a a sequence of finite Galois extensions for each $\alpha \in \Delta$ then we have $\left(E_{\Delta}^{\prime \prime+}\right)^{H_{\Delta}^{\prime}}=E_{\Delta}^{\prime+}$. The containment $\left(E_{\Delta}^{\prime \prime+}\right)^{H_{\Delta}^{\prime}} \supseteq E_{\Delta}^{\prime+}$ is clear. We prove the converse by induction on $|\Delta|$. Note that the ideal $\mathcal{M}_{\alpha} \triangleleft E_{\Delta}^{\prime \prime+}$ generated by $X_{\alpha}^{\prime \prime}$ is invariant under the action of $H_{\Delta}^{\prime}$ for any fixed $\alpha$ in $\Delta$. Moreover, for any integer $k \geq 1$ the ring $E_{\alpha}^{\prime \prime+} / \mathcal{M}_{\alpha}^{k}$ is finite dimensional over $\mathbb{F}_{p}$. Therefore the image of $\left(E_{\Delta}^{\prime \prime+}\right)^{H_{\Delta}^{\prime}}$ under the quotient map $E_{\Delta}^{\prime \prime+} \rightarrow E_{\Delta}^{\prime \prime+} / \mathcal{M}_{\alpha}^{k}$ is contained in

$$
\begin{aligned}
\left(E_{\Delta}^{\prime \prime+} / \mathcal{M}_{\alpha}^{k}\right)^{H_{\Delta}^{\prime}} \subseteq\left(E_{\Delta}^{\prime \prime+} / \mathcal{M}_{\alpha}^{k}\right)^{H_{\Delta \backslash\{\alpha\}}^{\prime}}=\left(E_{\Delta \backslash\{\alpha\}}^{\prime \prime+} \otimes_{\mathbb{F}_{p}}\left(E_{\alpha}^{\prime \prime+} / \mathcal{M}_{\alpha}^{k}\right)\right)^{H_{\Delta \backslash\{\alpha\}}^{\prime}}= \\
=\left(E_{\Delta \backslash\{\alpha\}}^{\prime \prime+}\right)^{H_{\Delta \backslash\{\alpha\}}^{\prime}} \otimes_{\mathbb{F}_{p}}\left(E_{\alpha}^{\prime \prime+} / \mathcal{M}_{\alpha}^{k}\right)=E_{\Delta \backslash\{\alpha\}}^{\prime+} \otimes_{\mathbb{F}_{p}}\left(E_{\alpha}^{\prime \prime+} / \mathcal{M}_{\alpha}^{k}\right)
\end{aligned}
$$


by induction. Taking the projective limit with respect to $k \geq 1$ we deduce that $\left(E_{\Delta}^{\prime \prime+}\right)^{H_{\Delta}^{\prime}}$ is contained in the power series ring

$$
\left(\mathbb{F}_{q_{\alpha}^{\prime \prime}} \otimes_{\mathbb{F}_{p}} \bigotimes_{\beta \in \Delta \backslash\{\alpha\}, \mathbb{F}_{p}} \mathbb{F}_{q_{\beta}^{\prime}}\right)\left[\left[X_{\alpha}^{\prime \prime}, X_{\beta}^{\prime} \mid \beta \in \Delta \backslash\{\alpha\}\right]\right] \subseteq E_{\Delta}^{\prime \prime+}
$$

Now using the action of $H_{\alpha}^{\prime}$ in a similar argument as above (reducing modulo the $k$ th power of the ideal generated by all the $X_{\beta}^{\prime}, \beta \in \Delta \backslash\{\alpha\}$ for all $k \geq 1$ ) we deduce the statement.

The subring $E_{\Delta, \circ}^{s e p} \cong \bigotimes_{\alpha \in \Delta, \mathbb{F}_{p}} E_{\alpha}^{s e p}$ in $E_{\Delta}^{s e p}$ is the inductive limit of $E_{\Delta, \circ}^{\prime} \subseteq E_{\Delta}^{\prime}$ where $E_{\alpha}^{\prime}$ runs through the finite separable extensions of $E_{\alpha}$ for each $\alpha \in \Delta$.

Let $V$ be a finite dimensional representation of the group $G_{\mathbb{Q}_{p}, \Delta}$ over $\mathbb{F}_{p}$. The basechange $E_{\Delta}^{s e p} \otimes_{\mathbb{F}_{p}} V$ is equipped with the diagonal semilinear action of $G_{\mathbb{Q}_{p}, \Delta}$ and with the Frobenii $\varphi_{\alpha}$ for $\alpha \in \Delta$. These all commute with each other. We define the value of the functor $\mathbb{D}$ at $V$ by putting

$$
\mathbb{D}(V):=\left(E_{\Delta}^{\text {sep }} \otimes_{\mathbb{F}_{p}} V\right)^{H_{\mathbb{Q}_{p}, \Delta}} .
$$

By Lemma $3.3 \mathbb{D}(V)$ is a module over $E_{\Delta}$ inheriting the action of the monoid $T_{+, \Delta}$ from the action of $\varphi_{\alpha}(\alpha \in \Delta)$ and the Galois group $G_{\mathbb{Q}_{p}, \Delta}$ on $E_{\Delta}^{s e p} \otimes_{\mathbb{F}_{p}} V$. Our key Lemma is the following.

Lemma 3.4. The $E_{\Delta}^{s e p}$-module $E_{\Delta}^{s e p} \otimes_{\mathbb{F}_{p}} V$ admits a basis consisting of elements fixed by $H_{\mathbb{Q}_{p}, \Delta}$.

Proof. At first consider the $E_{\Delta, o}^{s e p}$-module $E_{\Delta, \circ}^{s e p} \otimes_{\mathbb{F}_{p}} V$. We show by induction on $|\Delta|$ that $E_{\Delta, o}^{s e p} \otimes_{\mathbb{F}_{p}} V$ admits a basis consisting of $H_{\mathbb{Q}_{p}, \Delta}$-invariant vectors. The statement follows from this noting that $E_{\Delta, \circ}^{s e p}$ is a subring in $E_{\Delta}^{s e p}$ therefore the required basis exists also in $E_{\Delta}^{s e p} \otimes_{\mathbb{F}_{p}} V \cong$ $E_{\Delta}^{s e p} \otimes_{E_{\Delta}^{s e p}}\left(E_{\Delta, o}^{s e p} \otimes_{\mathbb{F}_{p}} V\right)$.

By Hilbert's Thm. 90 the $H_{\mathbb{Q}_{p}, \alpha}$-module $E_{\alpha}^{s e p} \otimes_{\mathbb{F}_{p}} V$ is trivial for each $\alpha \in \Delta$. So we have an $E_{\alpha}^{s e p}$-basis $e_{1}^{(\alpha)}, \ldots, e_{d}^{(\alpha)}$ of $E_{\alpha}^{s e p} \otimes_{\mathbb{F}_{p}} V$ consisting of $H_{\mathbb{Q}_{p}, \alpha}$-invariant elements. Since we have an action of the direct product $H_{\mathbb{Q}_{p}, \Delta}$ on $V$, the $E_{\alpha}$-vector space

$$
V_{\alpha}:=E_{\alpha} e_{1}^{(\alpha)}+\cdots+E_{\alpha} e_{d}^{(\alpha)}=\left(E_{\alpha}^{s e p} \otimes_{\mathbb{F}_{p}} V\right)^{H_{\mathbb{Q}_{p}, \alpha}}
$$

admits a linear action of the group $H_{\mathbb{Q}_{p}, \Delta \backslash\{\alpha\}}$. Now note that the representations $V$ and $V_{\alpha}$ of the group $H_{\mathbb{Q}_{p}, \Delta \backslash\{\alpha\}}$ become isomorphic over the field $E_{\alpha}^{s e p}$ by construction. Since $H_{\mathbb{Q}_{p}, \Delta \backslash\{\alpha\}}$ acts through a finite quotient on $V$, there is a finite extension $E_{\alpha}^{\prime}$ of $E_{\alpha}$ contained in $E_{\alpha}^{s e p}$ such that we have an isomorphism $E_{\alpha}^{\prime} \otimes_{\mathbb{F}_{p}} V \cong E_{\alpha}^{\prime} \otimes_{E_{\alpha}} V_{\alpha}$ of $H_{\mathbb{Q}_{p}, \Delta \backslash\{\alpha\}}$-representations. Making this identification and writing $e_{i}:=1 \otimes e_{i} \in E_{\alpha}^{\prime} \otimes_{\mathbb{F}_{p}} V\left(\right.$ resp. $\left.e_{i}^{(\alpha)}:=1 \otimes e_{i}^{(\alpha)}\right), i=1, \ldots, d$, for a basis $e_{1}, \ldots, e_{d}$ in $V$ (resp. for the basis $e_{1}^{(\alpha)}, \ldots e_{d}^{(\alpha)}$ in $V_{\alpha}$ ) by an abuse of notation, we find a matrix $B \in \mathrm{GL}_{d}\left(E_{\alpha}^{\prime}\right)$ with $B \rho(h)=\rho_{\alpha}(h) B$ for all $h \in H_{\mathbb{Q}_{p}, \Delta \backslash\{\alpha\}}$ where $\rho(h) \in \mathrm{GL}_{d}\left(\mathbb{F}_{p}\right)$ (resp. $\rho_{\alpha}(h) \in \mathrm{GL}_{d}\left(E_{\alpha}\right)$ ) is the matrix of the action of $h$ on $V$ (resp. on $V_{\alpha}$ ) in the basis $e_{1}, \ldots, e_{d}$ (resp. $\left.e_{1}^{(\alpha)}, \ldots e_{d}^{(\alpha)}\right)$. Now $E_{\alpha}^{\prime} / E_{\alpha}$ is a finite separable extension, so there exists a primitive element $u \in E_{\alpha}^{\prime}$ with $E_{\alpha}^{\prime}=E_{\alpha}(u)$. Hence we may write $B$ is a sum $B=B(u)=$ $B_{0}+B_{1} u+\cdots+B_{n-1} u^{n-1}$ for some matrices $B_{0}, B_{1}, \ldots, B_{n-1} \in E_{\alpha}^{d \times d}$ with $n:=\left|E_{\alpha}^{\prime}: E_{\alpha}\right|$. Since $\operatorname{det} B \neq 0$, the polynomial $\operatorname{det}(B(x)):=\operatorname{det}\left(B_{0}+B_{1} x+\cdots+B_{n-1} x^{n-1}\right) \in E_{\alpha}[x]$ is not identically 0 . As $E_{\alpha}$ is an infinite field, there exists a $u_{0} \in E_{\alpha}$ with $\operatorname{det} B\left(u_{0}\right) \neq 0$. Now 
we have $\rho(h)=B\left(u_{0}\right)^{-1} \rho_{\alpha}(h) B\left(u_{0}\right)$ for all $h \in H_{\mathbb{Q}_{p}, \Delta \backslash\{\alpha\}}$, ie. the representations $V$ and $V_{\alpha}$ of $H_{\mathbb{Q}_{p}, \Delta \backslash\{\alpha\}}$ are isomorphic already over $E_{\alpha}$. This shows that there exists a basis $v_{1}^{(\alpha)}, \ldots v_{d}^{(\alpha)}$ in $V_{\alpha}$ such that the action of each $h$ in $H_{\mathbb{Q}_{p}, \Delta \backslash\{\alpha\}}$ is given by a matrix in $\mathrm{GL}_{d}\left(\mathbb{F}_{p}\right)$ in this basis. We put

$$
\begin{aligned}
& V_{\Delta \backslash\{\alpha\}}:=\mathbb{F}_{p} v_{1}^{(\alpha)}+\ldots \mathbb{F}_{p} v_{d}^{(\alpha)} \subset V_{\alpha}=\left(E_{\alpha}^{s e p} \otimes_{\mathbb{F}_{p}} V\right)^{H_{\mathbb{Q}_{p}, \alpha}}= \\
= & \left(\bigotimes_{\beta \in \Delta \backslash\{\alpha\}} 1 \otimes\left(E_{\alpha}^{s e p} \otimes_{\mathbb{F}_{p}} V\right)\right)^{H_{\mathbb{Q}_{p}, \alpha}} \subseteq\left(E_{\Delta, \circ}^{s e p} \otimes_{\mathbb{F}_{p}} V\right)^{H_{\mathbb{Q}_{p}, \alpha}} .
\end{aligned}
$$

By induction we find a basis $v_{1}, \ldots, v_{n}$ of $E_{\Delta \backslash\{\alpha\}}^{s e p} \otimes_{\mathbb{F}_{p}} V_{\Delta \backslash\{\alpha\}} \subseteq\left(E_{\Delta, \circ}^{s e p} \otimes_{\mathbb{F}_{p}} V\right)^{H_{\mathbb{Q}_{p}, \alpha}}$ consisting of $H_{\mathbb{Q}_{p}, \Delta \backslash\{\alpha\}}$-invariant elements which are $H_{\mathbb{Q}_{p}, \alpha}$-invariant, as well, by construction. Therefore $v_{1}, \ldots, v_{n}$ is an $H_{\mathbb{Q}_{p}, \Delta}$-invariant basis of $E_{\Delta, o}^{s e p} \otimes_{\mathbb{F}_{p}} V$ as required.

Lemma 3.5. We have $\left(E_{\Delta}^{\text {sep }}\right)^{\times} \cap E_{\Delta}=E_{\Delta}^{\times}$.

Proof. Let $u$ be arbitrary in $\left(E_{\Delta}^{s e p}\right)^{\times} \cap E_{\Delta}$. Since $u$ is invariant under the action of $H_{\mathbb{Q}_{p}, \Delta}$, so is its inverse $u^{-1}$ whence it also lies in $E_{\Delta}$ by Lemma 3.3 .

Lemma 3.6. We have $\bigcap_{\alpha \in \Delta}\left(E_{\Delta}^{s e p}\right)^{\varphi_{\alpha}=\mathrm{id}}=\mathbb{F}_{p}$.

Proof. The containment $\mathbb{F}_{p} \subseteq \bigcap_{\alpha \in \Delta}\left(E_{\Delta}^{s e p}\right)^{\varphi_{\alpha}=\mathrm{id}} \subseteq\left(E_{\Delta}^{s e p}\right)^{\varphi_{s}=\mathrm{id}}$ is obvious. On the other hand, let $u \in E_{\Delta}^{s e p}$ be arbitrary such that $\varphi_{\alpha}(u)=u$ for all $\alpha \in \Delta$. Then we also have $u^{p}=\varphi_{s}(u)=u$ as $\varphi_{s}$ is the absolute Frobenius on $E_{\Delta}^{s e p}$. Since $E_{\Delta}^{s e p}$ is defined as an inductive limit, $u$ lies in $E_{\Delta}^{\prime} \cong\left(\bigotimes_{\alpha \in \Delta, \mathbb{F}_{p}} \mathbb{F}_{q_{\alpha}}\right)\left[\left[X_{\alpha}^{\prime} \mid \alpha \in \Delta\right]\right]\left[X_{\Delta}\right]$ for some collection $E_{\alpha}^{\prime}=\mathbb{F}_{q_{\alpha}}\left(\left(X_{\alpha}^{\prime}\right)\right)(\alpha \in \Delta)$ of finite separable extensions of $E_{\alpha}$. Note that $\bigotimes_{\alpha \in \Delta, \mathbb{F}_{p}} \mathbb{F}_{q_{\alpha}}$ is a finite étale algebra over $\mathbb{F}_{p}$, in particular, it is reduced. Therefore we have $\left|u^{p}\right|_{\text {prod }}=|u|_{\text {prod }}^{p}$. We deduce $|u|_{\text {prod }}=1$ unless $u=0$. In particular, $u$ lies in $E_{\Delta}^{\prime+}=\left(\bigotimes_{\alpha \in \Delta, \mathbb{F}_{p}} \mathbb{F}_{q_{\alpha}}\right)\left[\left[X_{\alpha}^{\prime} \mid \alpha \in \Delta\right]\right]$. The constant term $u_{0} \in \bigotimes_{\alpha \in \Delta, \mathbb{F}_{p}} \mathbb{F}_{q_{\alpha}}$ also satisfies $\varphi_{\alpha}\left(u_{0}\right)=u_{0}$ for all $\alpha \in \Delta$. For a fixed $\alpha \in \Delta$ we choose an $\mathbb{F}_{p \text {-basis }} d_{1}, \ldots, d_{n}$ of $\bigotimes_{\beta \in \Delta \backslash\{\alpha\}, \mathbb{F}_{p}} \mathbb{F}_{q_{\beta}}$ and write $u_{0}=\sum_{i=1}^{n} c_{i} \otimes d_{i}$ with $c_{i} \in \mathbb{F}_{q_{\alpha}}$. This decomposition is unique and we compute

$$
\sum_{i=1}^{n} c_{i} \otimes d_{i}=u_{0}=\varphi_{\alpha}\left(u_{0}\right)=\sum_{i=1}^{n} c_{i}^{p} \otimes d_{i} .
$$

We deduce $c_{i}=c_{i}^{p}$, ie. $c_{i} \in \mathbb{F}_{p}$ for all $1 \leq i \leq n$. It follows by induction on $|\Delta|$ that $u_{0}$ lies in $\mathbb{F}_{p}$. Now $u-u_{0}$ is also fixed by each $\varphi_{\alpha}(\alpha \in \Delta)$, but we have $\left|u-u_{0}\right|_{\text {prod }}<1$. This implies by the discussion above that $u=u_{0}$ is in $\mathbb{F}_{p}$ as desired.

Proposition 3.7. $\mathbb{D}(V)$ is an étale $T_{+, \Delta}$-module over $E_{\Delta}$ of rank $d:=\operatorname{dim}_{\mathbb{F}_{p}} V$. Moreover, we have $E_{\Delta}^{\text {sep }} \otimes_{E_{\Delta}} \mathbb{D}(V) \cong E_{\Delta}^{\text {sep }} \otimes_{\mathbb{F}_{p}} V$ and

$$
V=\bigcap_{\alpha \in \Delta}\left(E_{\Delta}^{s e p} \otimes_{E_{\Delta}} \mathbb{D}(V)\right)^{\varphi_{\alpha}=\mathrm{id}} .
$$

Proof. By Lemmata 3.3 and $3.4 \mathbb{D}(V)$ is a free module of rank $d$ over $E_{\Delta}$. Moreover, the matrix of $\varphi_{\alpha}$ in any basis of $\mathbb{D}(V)$ is invertible in $E_{\Delta}^{s e p}$, therefore also in $E_{\Delta}$ by Lemma 3.5 . So the action of $T_{+, \Delta}$ on $\mathbb{D}(V)$ is étale. The last statement is a direct consequence of Lemmata 3.4 and 3.6 . 
Lemma 3.8. For objects $V, V_{1}, V_{2}$ in $\operatorname{Rep}_{\mathbb{F}_{p}}\left(G_{\mathbb{Q}_{p}, \Delta}\right)$ we have $\mathbb{D}\left(V_{1} \otimes_{\mathbb{F}_{p}} V_{2}\right) \cong \mathbb{D}\left(V_{1}\right) \otimes_{E_{\Delta}} \mathbb{D}\left(V_{2}\right)$ and $\mathbb{D}\left(V^{*}\right) \cong \mathbb{D}(V)^{*}$.

Proof. We compute

$$
\begin{aligned}
& \mathbb{D}\left(V_{1} \otimes_{\mathbb{F}_{p}} V_{2}\right)=\left(E_{\Delta}^{\text {sep }} \otimes_{\mathbb{F}_{p}} V_{1} \otimes_{\mathbb{F}_{p}} V_{2}\right)^{H_{\mathbb{Q}_{p}, \Delta}} \cong\left(\left(E_{\Delta}^{\text {sep }} \otimes_{\mathbb{F}_{p}} V_{1}\right) \otimes_{E_{\Delta}^{\text {sep }}}\left(E_{\Delta}^{\text {sep }} \otimes_{\mathbb{F}_{p}} V_{2}\right)\right)^{H_{\mathbb{Q}_{p}, \Delta}} \cong \\
& \left(\left(E_{\Delta}^{\text {sep }} \otimes_{E_{\Delta}} \mathbb{D}\left(V_{1}\right)\right) \otimes_{E_{\Delta}^{\text {sep }}}\left(E_{\Delta}^{\text {sep }} \otimes_{E_{\Delta}} \mathbb{D}\left(V_{2}\right)\right)\right)^{H_{\mathbb{Q}_{p}, \Delta}} \cong \\
& \cong\left(E_{\Delta}^{s e p} \otimes_{E_{\Delta}}\left(\mathbb{D}\left(V_{1}\right) \otimes_{E_{\Delta}} \mathbb{D}\left(V_{2}\right)\right)\right)^{H_{\mathbb{Q}_{p}, \Delta}} \cong \mathbb{D}\left(V_{1}\right) \otimes_{E_{\Delta}} \mathbb{D}\left(V_{2}\right) \text {. }
\end{aligned}
$$

For the second statement we have

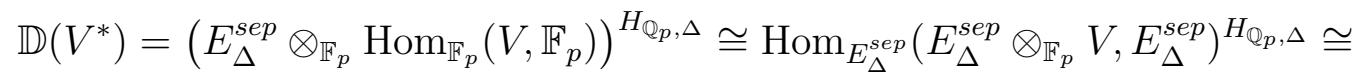

$$
\begin{aligned}
& \cong \operatorname{Hom}_{E_{\Delta}^{s e p}}\left(E_{\Delta}^{s e p} \otimes_{E_{\Delta}} \mathbb{D}(V), E_{\Delta}^{\text {sep }}\right)^{H_{\mathbb{Q}_{p}, \Delta}} \cong\left(E_{\Delta}^{\text {sep }} \otimes_{E_{\Delta}} \operatorname{Hom}_{E_{\Delta}}\left(\mathbb{D}(V), E_{\Delta}\right)\right)^{H_{\mathbb{Q}_{p}, \Delta}} \cong \mathbb{D}(V)^{*}
\end{aligned}
$$

Theorem 3.9. $\mathbb{D}$ is a fully faithful tensor functor from the category $\operatorname{Rep}_{\mathbb{F}_{p}}\left(G_{\mathbb{Q}_{p}, \Delta}\right)$ to the category $\mathcal{D}^{e t}\left(\varphi_{\Delta}, \Gamma_{\Delta}, E_{\Delta}\right)$.

Proof. Let $f: V_{1} \rightarrow V_{2}$ be a nonzero morphism in $\operatorname{Rep}_{\mathbb{F}_{p}}\left(G_{\mathbb{Q}_{p}, \Delta}\right)$. Then the $E_{\Delta}^{s e p}$-linear map id $\otimes f: E_{\Delta}^{s e p} \otimes_{\mathbb{F}_{p}} V_{1} \rightarrow E_{\Delta}^{s e p} \otimes_{\mathbb{F}_{p}} V_{2}$ is also nonzero. By the last statement in Prop. 3.7 it follows that $\mathbb{D}(f) \neq 0$ therefore the faithfulness.

Now let $V_{1}$ and $V_{2}$ be arbitrary objects in $\operatorname{Rep}_{\mathbb{F}_{p}}\left(G_{\mathbb{Q}_{p}, \Delta}\right)$ and $\theta: \mathbb{D}\left(V_{1}\right) \rightarrow \mathbb{D}\left(V_{2}\right)$ be a morphism in $\mathcal{D}^{e t}\left(\varphi_{\Delta}, \Gamma_{\Delta}, E_{\Delta}\right)$. Then by Prop. 3.7 we obtain a $G_{\mathbb{Q}_{p}, \Delta^{-}}$-equivariant $\mathbb{F}_{p}$-linear map

$$
f: V_{1}=\bigcap_{\alpha \in \Delta}\left(E_{\Delta}^{s e p} \otimes_{E_{\Delta}} \mathbb{D}\left(V_{1}\right)\right)^{\varphi_{\alpha}=\mathrm{id}} \rightarrow \bigcap_{\alpha \in \Delta}\left(E_{\Delta}^{\text {sep }} \otimes_{E_{\Delta}} \mathbb{D}\left(V_{2}\right)\right)^{\varphi_{\alpha}=\mathrm{id}}=V_{2}
$$

induced by $\theta$ for which we have $\theta=\mathbb{D}(f)$. Therefore $\mathbb{D}$ is full. The compatibility with tensor products is proven in Lemma 3.8 .

Remark. Note that any étale $T_{+, \Delta}$-module $D$ in the image of the functor $\mathbb{D}$ is free as a module over $E_{\Delta}$ by construction.

Consider the diagonal embedding diag: $G_{\mathbb{Q}_{p}} \hookrightarrow G_{\mathbb{Q}_{p}, \Delta}$ sending $g \in G_{\mathbb{Q}_{p}}$ to $(g, \ldots, g)$. This defines a functor $\widehat{\operatorname{diag}}: \operatorname{Rep}_{\mathbb{F}_{p}}\left(G_{\mathbb{Q}_{p}, \Delta}\right) \rightarrow \operatorname{Rep}_{\mathbb{F}_{p}}\left(G_{\mathbb{Q}_{p}}\right)$ via restriction. On the other hand, we have the reduction map $\ell: \mathcal{D}^{e t}\left(\varphi_{\Delta}, \Gamma_{\Delta}, E_{\Delta}\right) \rightarrow \mathcal{D}^{e t}(\varphi, \Gamma, E)$ to usual $(\varphi, \Gamma)$-modules defined in section 2.4 of [11]. Recall that this is given by taking the quotient by the ideal generated by $\left(X_{\alpha}-X_{\beta} \mid \alpha, \beta \in \Delta\right)$ and restricting to the diagonal $\varphi=\varphi_{s}=\prod_{\alpha \in \Delta} \varphi_{\alpha}$ and $\Gamma:=$ $\{(\gamma, \ldots, \gamma)\} \leq \Gamma_{\Delta}$.

Corollary 3.10. There is a natural isomorphism $\widehat{\mathrm{diag}} \cong \mathbb{V}_{F} \circ \mathrm{lo \mathbb {D }}$ of functors $\operatorname{Rep}_{\mathbb{F}_{p}}\left(G_{\mathbb{Q}_{p}, \Delta}\right) \rightarrow$ $\operatorname{Rep}_{\mathbb{F}_{p}}\left(G_{\mathbb{Q}_{p}}\right)$ where $\mathbb{V}_{F}: \mathcal{D}^{\text {et }}(\varphi, \Gamma, E) \rightarrow \operatorname{Rep}_{\mathbb{F}_{p}}\left(G_{\mathbb{Q}_{p}}\right)$ is Fontaine's functor from classical étale $(\varphi, \Gamma)$-modules to Galois representations. 
Proof. We may identify $E_{\alpha} \stackrel{\sim}{\rightarrow} E=\mathbb{F}_{p}((X))$ by sending $X_{\alpha} \rightarrow X$ for all $\alpha \in \Delta$. We extend this identification to $E_{\alpha}^{s e p} \rightarrow E^{s e p}$. So we obtain a map $\ell^{\text {sep }}: E_{\Delta}^{s e p} \rightarrow E^{\text {sep }}$ sending each subring $E_{\alpha}^{s e p}$ to $E^{s e p}$ via these identifications and completing on the level of each finite extension $E_{\Delta}^{\prime}$. We do this in a way so that the diagonal embedding of $G_{\mathbb{Q}_{p}} \hookrightarrow G_{\mathbb{Q}_{p}, \Delta}$ acts on the quotient $E^{\text {sep }}$ in the usual way. The restriction of $\ell^{\text {sep }}$ to $E_{\Delta}$ is the map $\ell: E_{\Delta} \rightarrow E$ defined above, so the diagram

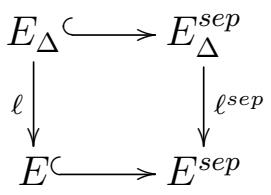

commutes. Thus for an object $V$ in $\operatorname{Rep}_{\mathbb{F}_{p}}\left(G_{\mathbb{Q}_{p}, \Delta}\right)$ we compute

$$
\begin{array}{r}
\mathbb{V}_{F} \circ \ell \circ \mathbb{D}(V)=\mathbb{V}_{F}\left(E \otimes_{E_{\Delta}, \ell} \mathbb{D}(V)\right)=\mathbb{V}_{F}\left(\left(E^{\text {sep }}\right)^{H_{\mathbb{Q}_{p}}} \otimes_{E_{\Delta}, \ell} \mathbb{D}(V)\right)= \\
=\mathbb{V}_{F}\left(\left(E^{\text {sep }} \otimes_{E_{\Delta}^{\text {sep }}, \ell^{\text {sep }}} E_{\Delta}^{\text {sep }} \otimes_{E_{\Delta}} \mathbb{D}(V)\right)^{H_{\mathbb{Q}_{p}}}\right)=\mathbb{V}_{F}\left(\left(E^{\text {sep }} \otimes_{E_{\Delta}^{\text {sep }}, \ell^{\text {sep }}} E_{\Delta}^{\text {sep }} \otimes_{\mathbb{F}_{p}} V\right)^{H_{\mathbb{Q}_{p}}}\right)= \\
=\mathbb{V}_{F}\left(\left(E^{\text {sep }} \otimes_{\mathbb{F}_{p}} V\right)^{H_{\mathbb{Q}_{p}}}\right)=\mathbb{V}_{F} \circ \mathbb{D}_{F}(V)=\left.V\right|_{\operatorname{diag}\left(G_{\mathbb{Q}_{p}}\right)}=\widehat{\operatorname{diag}}(V) .
\end{array}
$$

\subsection{The functor $\mathbb{V}$}

In order to show that the functor $\mathbb{D}$ is essentially surjective, we construct its quasi-inverse $\mathbb{V}$. Let $D$ be an object in $\mathcal{D}^{e t}\left(\varphi_{\Delta}, \Gamma_{\Delta}, E_{\Delta}\right)$. The group $G_{\mathbb{Q}_{p}, \Delta}$ acts on $E_{\Delta}^{\text {sep }} \otimes_{E_{\Delta}} D$ via the formula $g(\lambda \otimes x):=g(\lambda) \otimes \chi_{c y c}(g)(x)\left(g \in G_{\mathbb{Q}_{p}, \Delta}, \lambda \in E_{\Delta}^{s e p}, x \in D\right)$ where $\chi_{c y c}: G_{\mathbb{Q}_{p}, \Delta} \rightarrow \Gamma_{\Delta}$ is the quotient map. Moreover, each partial Frobenius $\varphi_{\alpha}(\alpha \in \Delta)$ acts semilinearly on $E_{\Delta}^{\text {sep }} \otimes_{E_{\Delta}} D$ via the formula $\varphi_{\alpha}(\lambda \otimes x):=\varphi_{\alpha}(\lambda) \otimes \varphi_{\alpha}(x)$. All these actions commute with each other by construction. We define

$$
\mathbb{V}(D):=\bigcap_{\alpha \in \Delta}\left(E_{\Delta}^{s e p} \otimes_{E_{\Delta}} D\right)^{\varphi_{\alpha}=\mathrm{id}}
$$

$\mathbb{V}(D)$ is a - a priori not necessarily finite dimensional-representation of $G_{\mathbb{Q}_{p}, \Delta}$ over $\mathbb{F}_{p}$.

Lemma 3.11. For any integer $r>0$ we have $\bigcap_{\beta \in \Delta}\left(E_{\Delta \backslash\{\alpha\}}^{\text {sep }}\left[X_{\alpha}\right] /\left(X_{\alpha}^{r}\right)\right)^{\varphi_{\beta}=\mathrm{id}}=\mathbb{F}_{p}\left[X_{\alpha}\right] /\left(X_{\alpha}^{r}\right)$.

Proof. This follows from Lemma 3.6 noting that $\mathbb{F}_{p}\left[X_{\alpha}\right] /\left(X_{\alpha}^{r}\right)$ is a finite dimensional $\mathbb{F}_{p^{-}}$ vector space on which $\varphi_{\beta}$ acts identically for all $\beta \in \Delta \backslash\{\alpha\}$ and we have $E_{\Delta \backslash\{\alpha\}}^{s e p}\left[X_{\alpha}\right] /\left(X_{\alpha}^{r}\right) \cong$ $E_{\Delta \backslash\{\alpha\}}^{s e p} \otimes_{\mathbb{F}_{p}} \mathbb{F}_{p}\left[X_{\alpha}\right] /\left(X_{\alpha}^{r}\right)$

Lemma 3.12. For any integer $r>0$ and finitely generated $E_{\bar{\alpha}}^{+} /\left(X_{\alpha}^{r}\right)$-module $M$ we have an identification $E_{\Delta \backslash\{\alpha\}}^{s e p}\left[X_{\alpha}\right] /\left(X_{\alpha}^{r}\right) \otimes_{E_{\bar{\alpha}}^{+} /\left(X_{\alpha}^{r}\right)} M \cong E_{\Delta \backslash\{\alpha\}}^{s e p} \otimes_{E_{\Delta \backslash\{\alpha\}}} M$.

Proof. This follows from the isomorphism $E_{\bar{\alpha}}^{+} /\left(X_{\alpha}^{r}\right) \cong E_{\Delta \backslash\{\alpha\}}\left[X_{\alpha}\right] /\left(X_{\alpha}^{r}\right)$.

For a subset $S \subseteq \Delta$ we put $E_{S}^{s e p+}:=\lim _{\longrightarrow} E_{S}^{\prime+}$ so we have $E_{S}^{s e p}=E_{S}^{s e p+}\left[X_{S}^{-1}\right]$.

Lemma 3.13. $E_{S}^{\text {sep }}$ (resp. $E_{S}^{s e p+}$ ) is flat as a module over $E_{S}$ (resp. over $E_{S}^{+}$) for all $S \subseteq \Delta$. 
Proof. By construction, $E_{S}^{\prime}$ (resp. $E_{S}^{++}$) is finite free over $E_{S}$ (resp. over $E_{S}^{+}$), so $E_{S}^{s e p}$ (resp. $\left.E_{S}^{s e p+}\right)$ is the direct limit of flat modules hence flat.

Lemma 3.14. We have $\left(E_{\Delta \backslash\{\alpha\}}^{\text {sep }+}\left[\left[X_{\alpha}\right]\right]\left[X_{\Delta}^{-1}\right]\right)^{H_{\mathbb{Q}_{p}, \Delta \backslash\{\alpha\}}}=E_{\Delta}$.

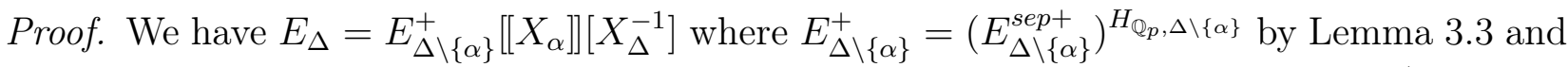
$H_{\mathbb{Q}_{p}, \Delta \backslash\{\alpha\}}$ acts trivially on both $X_{\alpha}$ and $X_{\Delta}$, so acts on the power series ring $E_{\Delta \backslash\{\alpha\}}^{+}\left[\left[X_{\alpha}\right]\right]$ coefficientwise.

Our main result in this section is the following

Theorem 3.15. The functors $\mathbb{D}$ and $\mathbb{V}$ are quasi-inverse equivalences of categories between the Tannakian categories $\operatorname{Rep}_{\mathbb{F}_{p}}\left(G_{\mathbb{Q}_{p}, \Delta}\right)$ and $\mathcal{D}^{e t}\left(\varphi_{\Delta}, \Gamma_{\Delta}, E_{\Delta}\right)$.

Corollary 3.16. Any object $D$ in $\mathcal{D}^{e t}\left(\varphi_{\Delta}, \Gamma_{\Delta}, E_{\Delta}\right)$ is a free module over $E_{\Delta}$.

Proof. This follows from the essential surjectivity of $\mathbb{D}$ using the remark after Thm. 3.9 .

Proof of Thm. 3.15. This is a long proof that we divide into 5 steps.

Step 1. Reducing the statement to the essential surjectivity of $\mathbb{D}$. By Thm. 3.9 the functor $\mathbb{D}$ is fully faithful and we have $\mathbb{V} \circ \mathbb{D}(V) \cong V$ naturally in $V$ for any object $V$ in $\operatorname{Rep}_{\mathbb{F}_{p}}\left(G_{\mathbb{Q}_{p}, \Delta}\right)$ by Prop. 3.7. Moreover, by Lemma $3.8 \mathbb{D}$ is compatible with tensor products and duals. So it remains to show that $\mathbb{D}$ is essentially surjective. We proceed by induction on $|\Delta|$. For $|\Delta|=1$ this is a classical result of Fontaine (see e.g. Thm. 2.21 in [5]). Suppose that $|\Delta|>1$, fix $\alpha \in \Delta$, and pick an object $D$ in $\mathcal{D}^{e t}\left(\varphi_{\Delta}, \Gamma_{\Delta}, E_{\Delta}\right)$.

Step 2. The goal here is to trivialize the $\varphi_{\beta}$-action $(\beta \in \Delta \backslash\{\alpha\})$ on $D_{\bar{\alpha}}^{+*} / X_{\alpha}^{r}$ uniformly in

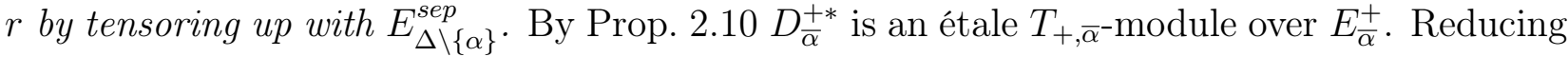
mod $X_{\alpha}^{r}$ for an integer $r>0$ we deduce that $D_{\bar{\alpha}, r}^{+*}:=D_{\bar{\alpha}}^{+*} / X_{\alpha}^{r} D_{\bar{\alpha}}^{+*}$ is an étale $T_{+, \bar{\alpha}^{-} \text {-module }}$ over $E_{\bar{\alpha}}^{+} /\left(X_{\alpha}^{r}\right) \cong E_{\Delta \backslash\{\alpha\}}\left[X_{\alpha}\right] /\left(X_{\alpha}^{r}\right)$. Since each $\varphi_{\beta}(\beta \in \Delta \backslash\{\alpha\})$ acts trivially on the variable $X_{\alpha}$, we have a natural isomorphism of functors

$$
E_{\Delta \backslash\{\alpha\}}\left[X_{\alpha}\right] /\left(X_{\alpha}^{r}\right) \otimes_{E_{\Delta \backslash\{\alpha\}}\left[X_{\alpha}\right] /\left(X_{\alpha}^{r}\right), \varphi_{t}} \cong E_{\Delta \backslash\{\alpha\}} \otimes_{E_{\Delta \backslash\{\alpha\}}, \varphi_{t}} \cdot
$$

for all $t \in T_{+, \bar{\alpha}}$. Hence $D_{\bar{\alpha}, r}^{+*}$ is an object in $\mathcal{D}^{e t}\left(\varphi_{\Delta \backslash\{\alpha\}}, \Gamma_{\Delta \backslash\{\alpha\}}, E_{\Delta \backslash\{\alpha\}}\right)$ since $E_{\Delta \backslash\{\alpha\}}\left[X_{\alpha}\right] /\left(X_{\alpha}^{r}\right)$ is finitely generated as a module over $E_{\Delta \backslash\{\alpha\}}$. By induction, we can trivialize $D_{\bar{\alpha}, r}^{+*}$ over $E_{\Delta \backslash\{\alpha\}}^{s e p}\left[X_{\alpha}\right] /\left(X_{\alpha}^{r}\right)$ : the natural map

$$
\begin{aligned}
E_{\Delta \backslash\{\alpha\}}^{s e p}\left[X_{\alpha}\right] /\left(X_{\alpha}^{r}\right) \otimes_{\mathbb{F}_{p}\left[X_{\alpha}\right] /\left(X_{\alpha}^{r}\right)} \bigcap_{\beta \in \Delta \backslash\{\alpha\}}\left(E_{\Delta \backslash\{\alpha\}}^{s e p}\left[X_{\alpha}\right] /\left(X_{\alpha}^{r}\right) \otimes_{E_{\bar{\alpha}}^{+} /\left(X_{\alpha}^{r}\right)} D_{\bar{\alpha}, r}^{+*}\right)^{\varphi_{\beta}=\mathrm{id}} \stackrel{\sim}{\rightarrow} \\
\stackrel{\sim}{\rightarrow} E_{\Delta \backslash\{\alpha\}}^{s e p}\left[X_{\alpha}\right] /\left(X_{\alpha}^{r}\right) \otimes_{E_{\bar{\alpha}}^{+} /\left(X_{\alpha}^{r}\right)} D_{\bar{\alpha}, r}^{+*} \cong E_{\Delta \backslash\{\alpha\}}^{s e p}\left[X_{\alpha}\right] /\left(X_{\alpha}^{r}\right) \otimes_{E_{\bar{\alpha}}^{+}} D_{\bar{\alpha}}^{+*}
\end{aligned}
$$

is an isomorphism for all $r>0$ using Lemmata 3.11 and 3.12. Our key Lemma is the following consequence of Prop. 2.10.

Lemma 3.17. There exists a finitely generated $E_{\Delta}^{+}$-submodule $M \leq D_{\bar{\alpha}}^{+*}$ such that

$$
\bigcap_{\beta \in \Delta \backslash\{\alpha\}}\left(E_{\Delta \backslash\{\alpha\}}^{s e p}\left[X_{\alpha}\right] /\left(X_{\alpha}^{r}\right) \otimes_{E_{\bar{\alpha}}^{+}} D_{\bar{\alpha},}^{+*}\right)^{\varphi_{\beta}=\mathrm{id}}
$$


is contained in the image of the map

$$
E_{\Delta \backslash\{\alpha\}}^{s e p+}\left[X_{\alpha}\right] /\left(X_{\alpha}^{r}\right) \otimes_{E_{\Delta}^{+}} M \rightarrow E_{\Delta \backslash\{\alpha\}}^{s e p+}\left[X_{\alpha}\right] /\left(X_{\alpha}^{r}\right) \otimes_{E_{\Delta}^{+}} D_{\bar{\alpha}}^{+*} \cong E_{\Delta \backslash\{\alpha\}}^{s e p}\left[X_{\alpha}\right] /\left(X_{\alpha}^{r}\right) \otimes_{E_{\bar{\alpha}}^{+}} D_{\bar{\alpha}}^{+*}
$$

induced by the inclusion $M \leq D_{\bar{\alpha}}^{+*}$ for all $r>0$. Moreover, $M$ can be chosen in such a way that (6) is injective.

Proof. We show that $M:=X_{\Delta \backslash\{\alpha\}}^{-k}\left(D^{+} \cap D_{\bar{\alpha}}^{+*}\right)$ will do for $k$ large enough. Since $D^{+}$is finitely generated over $E_{\Delta}^{+}$, so is $M$ by noetherianity. Using Lemma 2.11 we choose $k>0$ so that we have $D_{\bar{\alpha}}^{+*}=\bigcup_{l \geq 0} E_{\Delta}^{+} \varphi_{\bar{\alpha}}^{l}(M)$, ie. we put $M:=X_{\Delta \backslash\{\alpha\}}^{-1} D_{0}$. For any fixed $r>0$ there exists an integer $l_{r} \geq 0$ such that (5) is contained in

$$
\begin{array}{r}
E_{\Delta \backslash\{\alpha\}}^{s e p+}\left[X_{\alpha}\right] /\left(X_{\alpha}^{r}\right) \otimes_{E_{\Delta}^{+}} X_{\Delta \backslash\{\alpha\}}^{-p^{l_{r}}+1} M \subseteq E_{\Delta \backslash\{\alpha\}}^{s e p+}\left[X_{\alpha}\right] /\left(X_{\alpha}^{r}\right) \otimes_{E_{\Delta}^{+}} E_{\Delta}^{+} \varphi_{\bar{\alpha}}^{l_{r}}(M)= \\
=E_{\Delta \backslash\{\alpha\}}^{s e p+}\left[X_{\alpha}\right] /\left(X_{\alpha}^{r}\right) \varphi_{\bar{\alpha}}^{l_{r}}\left(E_{\Delta \backslash\{\alpha\}}^{s e p+}\left[X_{\alpha}\right] /\left(X_{\alpha}^{r}\right) \otimes_{E_{\Delta}^{+}} M\right)
\end{array}
$$

Now if $x$ lies in (5), then we have $\varphi_{\bar{\alpha}}^{l_{r}}(x)=x$. On the other hand, $x$ lies in

$$
E_{\Delta \backslash\{\alpha\}}^{\prime}\left[X_{\alpha}\right] /\left(X_{\alpha}^{r}\right) \varphi_{\bar{\alpha}}^{l_{r}}\left(E_{\Delta \backslash\{\alpha\}}^{\prime}\left[X_{\alpha}\right] /\left(X_{\alpha}^{r}\right) \otimes_{E_{\Delta}^{+}} M\right)
$$

for some finite separable extensions $E_{\beta}^{\prime} / E_{\beta}$ for $\beta \in \Delta \backslash\{\alpha\}$ and $E_{\Delta \backslash\{\alpha\}}^{\prime}:=\widehat{\bigotimes}_{\beta \in \Delta \backslash\{\alpha\}, \mathbb{F}_{p}} E_{\beta}^{\prime}$. Therefore $x$ lies in fact in $E_{\Delta \backslash\{\alpha\}}^{\prime}\left[X_{\alpha}\right] /\left(X_{\alpha}^{r}\right) \otimes_{E_{\Delta}^{+}} M$ by the injectivity of the map

$$
\begin{aligned}
\mathrm{id} \otimes \varphi_{\bar{\alpha}}^{l_{r}}: E_{\Delta \backslash\{\alpha\}}^{\prime}\left[X_{\alpha}\right] /\left(X_{\alpha}^{r}\right) \otimes_{E_{\Delta \backslash\{\alpha\}}^{\prime}\left[X_{\alpha}\right] /\left(X_{\alpha}^{r}\right), \varphi_{\frac{\bar{\alpha}}{\alpha}}^{l_{r}}}\left(E_{\Delta \backslash\{\alpha\}}^{\prime}\left[X_{\alpha}\right] /\left(X_{\alpha}^{r}\right) \otimes_{E_{\bar{\alpha}}^{+}} D_{\bar{\alpha}}^{+*}\right) \rightarrow \\
\rightarrow E_{\Delta \backslash\{\alpha\}}^{\prime}\left[X_{\alpha}\right] /\left(X_{\alpha}^{r}\right) \otimes_{E_{\bar{\alpha}}^{+}} D_{\bar{\alpha}}^{+*}
\end{aligned}
$$

( $D_{\bar{\alpha}}^{+*}$ is étale) noting that the absolute Frobenius $\varphi_{\bar{\alpha}}: E_{\Delta \backslash\{\alpha\}}^{\prime} \rightarrow E_{\Delta \backslash\{\alpha\}}^{\prime}$ is injective since the ring $E_{\Delta \backslash\{\alpha\}}^{\prime}$ is the localization of a power series ring over a finite étale algebra over $\mathbb{F}_{p}$, in particular, it is reduced.

Finally, by Lemma 2.7 $D_{\bar{\alpha}}^{+*} / M$ has no $X_{\alpha}$-torsion as $D_{\bar{\alpha}}^{+*} / M \cong D_{\bar{\alpha}}^{+*}+X_{\Delta \backslash\{\alpha\}}^{-k} D^{+} /\left(X_{\Delta \backslash\{\alpha\}}^{-k} D^{+}\right)$ is contained in $D_{\bar{\alpha}}^{+} /\left(X_{\Delta \backslash\{\alpha\}}^{-k} D^{+}\right) \cong D_{\bar{\alpha}}^{+} / D^{+}$. Therefore the map (6) is injective.

Step 3. The goal here is to show the following compatibility of our construction with projective limits with respect to $r$.

Lemma 3.18. We have

$$
\begin{aligned}
& {\underset{\lim }{r}}_{r}\left(E_{\Delta \backslash\{\alpha\}}^{s e p+}\left[X_{\alpha}\right] /\left(X_{\alpha}^{r}\right) \otimes_{E_{\Delta}^{+}} M\right) \cong E_{\Delta \backslash\{\alpha\}}^{s e p+}\left[\left[X_{\alpha}\right]\right] \otimes_{E_{\Delta}^{+}} M, \\
& {\underset{\lim }{r}}_{r}\left(E_{\Delta \backslash\{\alpha\}}^{s e p}\left[X_{\alpha}\right] /\left(X_{\alpha}^{r}\right) \otimes_{E_{\bar{\alpha}}^{+}} D_{\bar{\alpha}}^{+*}\right) \cong E_{\Delta \backslash\{\alpha\}}^{s e p}\left[\left[X_{\alpha}\right]\right] \otimes_{E_{\bar{\alpha}}^{+}} D_{\bar{\alpha}}^{+*} \quad \text {, and } \\
& \lim _{r}\left(E_{\Delta \backslash\{\alpha\}}^{s e p}\left[X_{\alpha}\right] /\left(X_{\alpha}^{r}\right) \otimes_{\mathbb{F}_{p}\left[X_{\alpha}\right] /\left(X_{\alpha}^{r}\right)} \bigcap_{\beta \in \Delta \backslash\{\alpha\}}\left(E_{\Delta \backslash\{\alpha\}}^{s e p}\left[X_{\alpha}\right] /\left(X_{\alpha}^{r}\right) \otimes_{E_{\bar{\alpha}}^{+} /\left(X_{\alpha}^{r}\right)} D_{\bar{\alpha}, r}^{+*}\right)^{\varphi_{\beta}=\mathrm{id}}\right) \cong \\
& \cong E_{\Delta \backslash\{\alpha\}}^{s e p}\left[\left[X_{\alpha}\right]\right] \otimes_{\mathbb{F}_{p} \llbracket X_{\alpha} \rrbracket} \bigcap_{\beta \in \Delta \backslash\{\alpha\}}\left(E_{\Delta \backslash\{\alpha\}}^{s e p}\left(\left(X_{\alpha}\right)\right) \otimes_{E_{\Delta}} D\right)^{\varphi_{\beta}=\mathrm{id}}
\end{aligned}
$$


Proof. Since $M$ is contained in $D, M$ has no $X_{\alpha}$-torsion. In particular, $M$ is flat as a module over the local ring $\mathbb{F}_{p}\left[\left[X_{\alpha}\right]\right]$. Now we deduce that $M$ and $E_{\Delta \backslash\{\alpha\}}^{s e p+}\left[X_{\alpha}\right] /\left(X_{\alpha}^{r}\right)$ are Tor-independent over $E_{\Delta}^{+}$by Lemma 3.13 since we have the identification

$$
E_{\Delta \backslash\{\alpha\}}^{s e p+}\left[X_{\alpha}\right] /\left(X_{\alpha}^{r}\right) \otimes_{E_{\Delta}^{+}} M \cong E_{\Delta \backslash\{\alpha\}}^{s e p+} \otimes_{E_{\Delta \backslash\{\alpha\}}^{+}}\left(\mathbb{F}_{p}\left[X_{\alpha}\right] /\left(X_{\alpha}^{r}\right) \otimes_{\mathbb{F}_{p} \llbracket X_{\alpha} \rrbracket} M\right) .
$$

On the other hand, $M$ is finitely generated over $E_{\Delta}^{+}$, so we short exact sequences

$$
0 \rightarrow M_{1} \rightarrow\left(E_{\Delta}^{+}\right)^{k_{0}} \stackrel{f_{0}}{\rightarrow} M \rightarrow 0 \quad \text { and } \quad 0 \rightarrow M_{2} \rightarrow\left(E_{\Delta}^{+}\right)^{k_{1}} \rightarrow M_{1} \rightarrow 0
$$

by noetherianity. In order to simplify notation write $(\cdot)_{r}$ for $E_{\Delta \backslash\{\alpha\}}^{s e p+}\left[X_{\alpha}\right] /\left(X_{\alpha}^{r}\right) \otimes_{E_{\Delta}^{+}} \cdot$ to obtain an exact sequence

$$
\left(M_{2}\right)_{r} \rightarrow\left(E_{\Delta}^{+}\right)_{r}^{k_{1}} \stackrel{f_{1, r}}{\rightarrow}\left(E_{\Delta}^{+}\right)_{r}^{k_{0}} \stackrel{f_{0, r}}{\rightarrow}(M)_{r} \rightarrow 0
$$

for all $r>0$ using the Tor-indepence above. Now since the natural map $(N)_{r_{1}} \rightarrow(N)_{r_{2}}$ is surjective for any $E_{\Delta}^{+}$-module $N$ and $r_{1} \geq r_{2}>0$ by the right exactness of $\cdot \otimes_{E_{\Delta}^{+}} N$, the natural map $\operatorname{Ker}\left(f_{0, r_{1}}\right) \rightarrow \operatorname{Ker}\left(f_{0, r_{2}}\right)$ is also surjective (applying this in case $N=M_{1}$ and a diagram chasing). So the Mittag-Leffler property is satisfied for these projective systems showing that the map $\lim _{r} f_{0, r}$ is surjective with kernel $\lim _{r} \operatorname{Ker}\left(f_{0, r}\right)=\lim _{r} \operatorname{Im}\left(f_{1, r}\right)$. Applying the same trick as above with $N=M_{2}$ we deduce that the projective system $\operatorname{Ker}\left(f_{1, r}\right)$ also satisfies the Mittag-Leffler property showing that $\lim _{r} f_{1, r}$ has image $\lim _{r} \operatorname{Im}\left(f_{1, r}\right)$. In particular, $\lim _{r}(M)_{r}$ is the cokernel of the map $\lim _{r} f_{1, r}:\left(E_{\Delta \backslash\{\alpha\}}^{s e p+}\left[\left[X_{\alpha}\right]\right]\right)^{k_{1}} \rightarrow\left(E_{\Delta \backslash\{\alpha\}}^{s e p+}\left[\left[X_{\alpha}\right]\right]\right)^{k_{0}}$ and so is $E_{\Delta \backslash\{\alpha\}}^{s e p+}\left[\left[X_{\alpha}\right]\right] \otimes_{E_{\Delta}^{+}} M$ as claimed. The second statement follows in the exactly same way.

For the third statement note that the isomorphism (4) and the surjectivity of the map $E_{\Delta \backslash\{\alpha\}}^{s e p}\left[X_{\alpha}\right] /\left(X_{\alpha}^{r_{1}}\right) \otimes_{E_{\bar{\alpha}}^{+}} D_{\bar{\alpha}}^{+*} \rightarrow E_{\Delta \backslash\{\alpha\}}^{s e p}\left[X_{\alpha}\right] /\left(X_{\alpha}^{r_{2}}\right) \otimes_{E_{\bar{\alpha}}^{+}} D_{\bar{\alpha}}^{+^{*}}$ implies that the map

$$
\begin{aligned}
& \bigcap_{\beta \in \Delta \backslash\{\alpha\}}\left(E_{\Delta \backslash\{\alpha\}}^{s e p}\left[X_{\alpha}\right] /\left(X_{\alpha}^{r_{1}}\right) \otimes_{E_{\bar{\alpha}}^{+} /\left(X_{\alpha}^{r_{1}}\right)} D_{\bar{\alpha}, r}^{+*}\right)^{\varphi_{\beta}=\mathrm{id}} \rightarrow \\
& \rightarrow \bigcap_{\beta \in \Delta \backslash\{\alpha\}}\left(E_{\Delta \backslash\{\alpha\}}^{s e p}\left[X_{\alpha}\right] /\left(X_{\alpha}^{r_{2}}\right) \otimes_{E_{\bar{\alpha}}^{+} /\left(X_{\alpha}^{r_{2}}\right)} D_{\bar{\alpha}, r}^{+*}\right)^{\varphi_{\beta}=\mathrm{id}}
\end{aligned}
$$

is also onto for all $r_{1} \geq r_{2}$. Therefore the natural map

$$
\begin{array}{r}
\bigcap_{\beta \in \Delta \backslash\{\alpha\}}\left(E_{\Delta \backslash\{\alpha\}}^{s e p}\left[\left[X_{\alpha}\right]\right] \otimes_{E_{\bar{\alpha}}^{+}} D_{\bar{\alpha}}^{+*}\right)^{\varphi_{\beta}=\mathrm{id}}= \\
=\underbrace{}_{r} \bigcap_{\beta \in \Delta \backslash\{\alpha\}}\left(E_{\Delta \backslash\{\alpha\}}^{s e p}\left[X_{\alpha}\right] /\left(X_{\alpha}^{r}\right) \otimes_{E_{\bar{\alpha}}^{+} /\left(X_{\alpha}^{r}\right)} D_{\bar{\alpha}, r}^{+*}\right)^{\varphi_{\beta}=\mathrm{id}} \rightarrow \\
\rightarrow \bigcap_{\beta \in \Delta \backslash\{\alpha\}}\left(E_{\Delta \backslash\{\alpha\}}^{s e p}\left[X_{\alpha}\right] /\left(X_{\alpha}\right) \otimes_{E_{\bar{\alpha}}^{+} /\left(X_{\alpha}\right)} D_{\bar{\alpha}, r}^{+*}\right)^{\varphi_{\beta}=\mathrm{id}}
\end{array}
$$


is also onto using the second statement of the Lemma. On the other hand, the kernel of this map equals

$$
\begin{array}{r}
\bigcap_{\beta \in \Delta \backslash\{\alpha\}}\left(E_{\Delta \backslash\{\alpha\}}^{s e p}\left[\left[X_{\alpha}\right]\right] \otimes_{E_{\bar{\alpha}}^{+}} D_{\bar{\alpha}}^{+*}\right)^{\varphi_{\beta}=\mathrm{id}} \cap X_{\alpha} E_{\Delta \backslash\{\alpha\}}^{s e p}\left[\left[X_{\alpha}\right]\right] \otimes_{E_{\bar{\alpha}}^{+}} D_{\bar{\alpha}}^{+*}= \\
=X_{\alpha} \bigcap_{\beta \in \Delta \backslash\{\alpha\}}\left(E_{\Delta \backslash\{\alpha\}}^{s e p}\left[\left[X_{\alpha}\right]\right] \otimes_{E_{\bar{\alpha}}^{+}} D_{\bar{\alpha}}^{+*}\right)^{\varphi_{\beta}=\mathrm{id}}
\end{array}
$$

since $X_{\alpha}$ is fixed by each $\varphi_{\beta}$ and $E_{\Delta \backslash\{\alpha\}}^{s e p}\left[\left[X_{\alpha}\right]\right] \otimes_{E_{\bar{\alpha}}^{+}} D_{\bar{\alpha}}^{+*}$ has no $X_{\alpha}$-torsion. This shows, in particular, that $\bigcap_{\beta \in \Delta \backslash\{\alpha\}}\left(E_{\Delta \backslash\{\alpha\}}^{s e p}\left[\left[X_{\alpha}\right]\right] \otimes_{E_{\bar{\alpha}}^{+}} D_{\bar{\alpha}}^{+*}\right)^{\varphi_{\beta}=\text { id }}$ is finitely generated over $\mathbb{F}_{p}\left[\left[X_{\alpha}\right]\right]$ by the topological Nakayama Lemma (see [1]). Moreover, it is torsion-free hence free as $E_{\Delta \backslash\{\alpha\}}^{s e p}\left[\left[X_{\alpha}\right]\right] \otimes_{E_{\bar{\alpha}}^{+}} D_{\bar{\alpha}}^{+*}$ has no $X_{\alpha}$-torsion either. In particular,

$$
E_{\Delta \backslash\{\alpha\}}^{s e p}\left[\left[X_{\alpha}\right]\right] \otimes_{\mathbb{F}_{p} \llbracket X_{\alpha} \rrbracket} \bigcap_{\beta \in \Delta \backslash\{\alpha\}}\left(E_{\Delta \backslash\{\alpha\}}^{s e p}\left(\left(X_{\alpha}\right)\right) \otimes_{E_{\Delta}} D\right)^{\varphi_{\beta}=\mathrm{id}}
$$

is $X_{\alpha}$-adically complete and the result follows.

Step 4. The goal here is to obtain a $\left(\varphi_{\alpha}, \Gamma_{\alpha}\right)$-module $D_{\alpha}$ over $E_{\alpha}$ (by trivializing the action of each $\left.\varphi_{\beta}, \beta \in \Delta \backslash\{\alpha\}\right)$ which is at the same time a linear representation of the group $G_{\mathbb{Q}_{p}, \Delta \backslash\{\alpha\}}$. We take projective limits of the inclusions in Lemma 3.17 with respect to $r$ to conclude (using Lemma 3.18) that

$$
\bigcap_{\beta \in \Delta \backslash\{\alpha\}}\left(E_{\Delta \backslash\{\alpha\}}^{s e p}\left[\left[X_{\alpha}\right]\right] \otimes_{E_{\bar{\alpha}}^{+}} D_{\bar{\alpha}}^{+*}\right)^{\varphi_{\beta}=\mathrm{id}}
$$

is contained in the image of the map

$$
E_{\Delta \backslash\{\alpha\}}^{s e p+}\left[\left[X_{\alpha}\right]\right] \otimes_{E_{\Delta}^{+}} M \rightarrow E_{\Delta \backslash\{\alpha\}}^{s e p}\left[\left[X_{\alpha}\right]\right] \otimes_{E_{\bar{\alpha}}^{+}} D_{\bar{\alpha}}^{+*} .
$$

Note that $M\left[X_{\Delta}^{-1}\right]=D_{\bar{\alpha}}^{+*}\left[X_{\Delta}^{-1}\right]=D_{\bar{\alpha}}^{+*}\left[X_{\alpha}^{-1}\right]=D$ and $\varphi_{\beta}$ acts trivially on $X_{\alpha}$. So inverting $X_{\Delta}$ above we deduce that

$$
D_{\alpha}:=\bigcap_{\beta \in \Delta \backslash\{\alpha\}}\left(E_{\Delta \backslash\{\alpha\}}^{s e p}\left(\left(X_{\alpha}\right)\right) \otimes_{E_{\Delta}} D\right)^{\varphi_{\beta}=\mathrm{id}}
$$

is contained in the image of the map

$$
E_{\Delta \backslash\{\alpha\}}^{s e p+}\left[\left[X_{\alpha}\right]\right]\left[X_{\Delta}^{-1}\right] \otimes_{E_{\Delta}} D \hookrightarrow E_{\Delta \backslash\{\alpha\}}^{s e p}\left(\left(X_{\alpha}\right)\right) \otimes_{E_{\Delta}} D
$$

On the other hand, by (4) and the third statement of Lemma 3.18 we have an isomorphism

$$
E_{\Delta \backslash\{\alpha\}}^{s e p}\left(\left(X_{\alpha}\right)\right) \otimes_{\mathbb{F}_{p}\left(\left(X_{\alpha}\right)\right)} D_{\alpha} \stackrel{\sim}{\rightarrow} E_{\Delta \backslash\{\alpha\}}^{s e p}\left(\left(X_{\alpha}\right)\right) \otimes_{E_{\Delta}} D
$$

Lemma 3.19. The finite dimensional $\mathbb{F}_{p}\left(\left(X_{\alpha}\right)\right)$-vector space $D_{\alpha}$ has the structure of an étale $\left(\varphi_{\alpha}, \Gamma_{\alpha}\right)$-module. At the same time it is a (linear) representation of the group $G_{\mathbb{Q}_{p}, \Delta \backslash\{\alpha\}}$. These two actions commute with each other. 
Proof. The operator $\varphi_{\alpha}$ and the groups $\Gamma_{\alpha}$ and $G_{\mathbb{Q}_{p}, \Delta \backslash\{\alpha\}}$ act naturally on $D_{\alpha}$. For the étaleness of the action of $\varphi_{\alpha}$ on $D_{\alpha}$ note that we have $\mathbb{F}_{p}\left(\left(X_{\alpha}\right)\right) \otimes_{\mathbb{F}_{p}\left(\left(X_{\alpha}\right)\right), \varphi_{\alpha}} D \cong D$ by the étale property of $\varphi_{\alpha}$ on $D$ and that $\varphi_{\beta}$ acts trivially on $\mathbb{F}_{p}\left(\left(X_{\alpha}\right)\right)$. So we compute

$$
\begin{aligned}
& \mathbb{F}_{p}\left(\left(X_{\alpha}\right)\right) \otimes_{\mathbb{F}_{p}\left(\left(X_{\alpha}\right)\right), \varphi_{\alpha}} D_{\alpha}=\mathbb{F}_{p}\left(\left(X_{\alpha}\right)\right) \otimes_{\mathbb{F}_{p}\left(\left(X_{\alpha}\right)\right), \varphi_{\alpha}} \bigcap_{\beta \in \Delta \backslash\{\alpha\}}\left(E_{\Delta \backslash\{\alpha\}}^{s e p}\left(\left(X_{\alpha}\right)\right) \otimes_{E_{\Delta}} D\right)^{\varphi_{\beta}=\mathrm{id}}= \\
&=\bigcap_{\beta \in \Delta \backslash\{\alpha\}}\left(\mathbb{F}_{p}\left(\left(X_{\alpha}\right)\right) \otimes_{\mathbb{F}_{p}\left(\left(X_{\alpha}\right)\right), \varphi_{\alpha}} E_{\Delta \backslash\{\alpha\}}^{s e p}\left(\left(X_{\alpha}\right)\right) \otimes_{E_{\Delta}} D\right)^{\varphi_{\beta}=\mathrm{id}}= \\
&=\bigcap_{\beta \in \Delta \backslash\{\alpha\}}\left(E_{\Delta \backslash\{\alpha\}}^{s e p}\left(\left(X_{\alpha}\right)\right) \otimes_{E_{\Delta}} \mathbb{F}_{p}\left(\left(X_{\alpha}\right)\right) \otimes_{\mathbb{F}_{p}\left(\left(X_{\alpha}\right)\right), \varphi_{\alpha}} D\right)^{\varphi_{\beta}=\mathrm{id}} \cong \\
& \cong \bigcap_{\beta \in \Delta \backslash\{\alpha\}}\left(E_{\Delta \backslash\{\alpha\}}^{s e p}\left(\left(X_{\alpha}\right)\right) \otimes_{E_{\Delta}} D\right)^{\varphi_{\beta}=\mathrm{id}}=D_{\alpha} .
\end{aligned}
$$

Step 5. We show the essential surjectivity of $\mathbb{D}$ here. Now we apply $\mathbb{V}_{F, \alpha}=\left(E_{\alpha}^{s e p} \otimes_{\mathbb{F}_{p}\left(\left(X_{\alpha}\right)\right)}\right.$ .) $\varphi_{\alpha}=$ id on $D_{\alpha}$ to obtain a finite dimensional $\mathbb{F}_{p}$-representation $V$ of $G_{\mathbb{Q}_{p}, \Delta}$. Moreover, we have $\operatorname{dim}_{\mathbb{F}_{p}} V=\operatorname{dim}_{\mathbb{F}_{p}\left(\left(X_{\alpha}\right)\right)} D_{\alpha}=\operatorname{rk}_{E_{\Delta}} D$ by the isomorphism (7) since $\mathbb{V}_{F, \alpha}$ is rank-preserving by Fontaine's classical result. Using again the isomorphism (7) and the containment $D_{\alpha} \subset$ $E_{\Delta \backslash\{\alpha\}}^{s e p+}\left[\left[X_{\alpha}\right]\right]\left[X_{\Delta}^{-1}\right] \otimes_{E_{\Delta}} D$ we conclude an injective map

$$
E_{\Delta \backslash\{\alpha\}}^{s e p+}\left[\left[X_{\alpha}\right]\right]\left[X_{\Delta}^{-1}\right] \otimes_{\mathbb{F}_{p}\left(\left(X_{\alpha}\right)\right)} D_{\alpha} \hookrightarrow E_{\Delta \backslash\{\alpha\}}^{s e p+}\left[\left[X_{\alpha}\right]\right]\left[X_{\Delta}^{-1}\right] \otimes_{E_{\Delta}} D
$$

and applying $E_{\alpha}^{\text {sep }} \otimes_{\mathbb{F}_{p}\left(\left(X_{\alpha}\right)\right)} \cdot$ another injective composite map

$$
\begin{array}{r}
E_{\Delta}^{s e p} \otimes_{\mathbb{F}_{p}} V \hookrightarrow \\
\hookrightarrow\left(E_{\Delta \backslash\{\alpha\}}^{s e p+}\left[\left[X_{\alpha}\right]\right]\left[X_{\Delta}^{-1}\right] \otimes_{\mathbb{F}_{p}\left(\left(X_{\alpha}\right)\right)} E_{\alpha}^{s e p}\right) \otimes_{\mathbb{F}_{p}} V \cong \\
\cong E_{\Delta \backslash\{\alpha\}}^{s e p+}\left[\left[X_{\alpha}\right]\right]\left[X_{\Delta}^{-1}\right] \otimes_{\mathbb{F}_{p}\left(\left(X_{\alpha}\right)\right)} E_{\alpha}^{s e p} \otimes_{\mathbb{F}_{p}\left(\left(X_{\alpha}\right)\right)} D_{\alpha}= \\
=E_{\alpha}^{s e p} \otimes_{\mathbb{F}_{p}\left(\left(X_{\alpha}\right)\right)} E_{\Delta \backslash\{\alpha\}}^{s e p+}\left[[ X _ { \alpha } ] \left[\left[X_{\Delta}^{-1}\right] \otimes_{\mathbb{F}_{p}\left(\left(X_{\alpha}\right)\right)} D_{\alpha} \hookrightarrow\right.\right. \\
\hookrightarrow\left(E_{\alpha}^{s e p} \otimes_{\mathbb{F}_{p}\left(\left(X_{\alpha}\right)\right)} E_{\Delta \backslash\{\alpha\}}^{s e p+}\left[\left[X_{\alpha}\right]\right]\left[X_{\Delta}^{-1}\right]\right) \otimes_{E_{\Delta}} D .
\end{array}
$$

Taking $G_{\mathbb{Q}_{p}, \Delta}$-invariants of this inclusion we deduce an inclusion $\mathbb{D}(V) \hookrightarrow D$ using Lemma 3.14. However, this is an isomorphism by Prop. 2.1 in [1] as $\mathbb{D}(V)$ and $D$ have the same rank.

Remarks. 1. Even though we have constructed $V$ in the proof of the above theorem by a different procedure from just putting $V:=\mathbb{V}(D)$, we still have an isomorphism $V \cong \mathbb{V}(\mathbb{D}(V)) \cong \mathbb{V}(D)$ by Prop. 3.7 .

2. If $\kappa$ is a finite extension of $\mathbb{F}_{p}$, then we have an equivalence of categories between $\operatorname{Rep}_{\kappa}\left(G_{\mathbb{Q}_{p}, \Delta}\right)$ and $\mathcal{D}^{e t}\left(\varphi_{\Delta}, \Gamma_{\Delta}, \kappa \otimes_{\mathbb{F}_{p}} E_{\Delta}\right)$. Indeed, we have a natural isomorphism $\left(\kappa \otimes_{\mathbb{F}_{p}}\right.$ $\left.E_{\Delta}^{s e p}\right) \otimes_{\kappa} \cdot \cong E_{\Delta}^{s e p} \otimes_{\mathbb{F}_{p}} \cdot$ as functors on $\operatorname{Rep}_{\kappa}\left(G_{\mathbb{Q}_{p}, \Delta}\right)$. 


\section{The case of $p$-adic representations}

\subsection{Cohomological preliminaries}

We will need the following multivariable analogue of Hilbert's Theorem 90 (additive form).

Proposition 4.1. The continuous group cohomology $H_{\text {cont }}^{1}\left(H_{\mathbb{Q}_{p}, \Delta}, E_{\Delta}^{\text {sep }}\right)$ vanishes.

Proof. By Prop. 3.3 it suffices to show that for finite Galois extensions $E_{\alpha}^{\prime} / E_{\alpha}$ (for all $\alpha \in \Delta$ ) with Galois group $H_{\alpha}^{\prime}:=\operatorname{Gal}\left(E_{\alpha}^{\prime} / E_{\alpha}\right)$ we have $H^{1}\left(H^{\prime}, E_{\Delta}^{\prime}\right)=\{1\}$ where we put $H^{\prime}:=$ $\prod_{\alpha \in \Delta} H_{\alpha}^{\prime}$. Choose a normal basis $e_{1}, \ldots, e_{n_{\alpha}} \in E_{\alpha}^{\prime}$ over $E_{\alpha}$ for each $\alpha \in \Delta$. By Lemma 3.2 the set $\left\{\prod_{\alpha \in \Delta} e_{i_{\alpha}} \mid 1 \leq i_{\alpha} \leq n_{\alpha}, \alpha \in \Delta\right\}$ is a basis of the free $E_{\Delta}$-module $E_{\Delta}^{\prime}$. In particular, $E_{\Delta}^{\prime} \cong E_{\Delta}\left[H^{\prime}\right]$ is induced as an $H^{\prime}$-module whence the cohomology group $H^{1}\left(H^{\prime}, E_{\Delta}^{\prime}\right)$ is trivial.

Let $D$ be an abelian group admitting an action of the commutative monoid $\prod_{\alpha \in \Delta} \varphi_{\alpha}^{\mathbb{N}}$. Fix a total ordering $<$ on $\Delta$ and consider the complex

$$
\Phi \bullet(D): 0 \rightarrow D \rightarrow \bigoplus_{\alpha \in \Delta} D \rightarrow \cdots \rightarrow \bigoplus_{\left\{\alpha_{1}, \ldots, \alpha_{r}\right\} \in\left(\begin{array}{c}
\Delta \\
r
\end{array}\right)} D \rightarrow \cdots \rightarrow D \rightarrow 0
$$

where for all $0 \leq r \leq|\Delta|-1$ the map $d_{\alpha_{1}, \ldots, \alpha_{r}}^{\beta_{1}, \ldots, \beta_{r+1}}: D \rightarrow D$ from the component in the $r$ th term corresponding to $\left\{\alpha_{1}, \ldots, \alpha_{r}\right\} \subseteq \Delta$ to the component corresponding to the $(r+1)$-tuple $\left\{\beta_{1}, \ldots, \beta_{r+1}\right\} \subseteq \Delta$ is given by

$$
d_{\alpha_{1}, \ldots, \alpha_{r}}^{\beta_{1}, \ldots, \beta_{r+1}}= \begin{cases}0 & \text { if }\left\{\alpha_{1}, \ldots, \alpha_{r}\right\} \nsubseteq\left\{\beta_{1}, \ldots, \beta_{r+1}\right\} \\ (-1)^{\varepsilon}\left(\mathrm{id}-\varphi_{\beta}\right) & \text { if }\left\{\beta_{1}, \ldots, \beta_{r+1}\right\}=\left\{\alpha_{1}, \ldots, \alpha_{r}\right\} \cup\{\beta\},\end{cases}
$$

where $\varepsilon=\varepsilon\left(\alpha_{1}, \ldots, \alpha_{r}, \beta\right)$ is the number of elements in the set $\left\{\alpha_{1}, \ldots, \alpha_{r}\right\}$ smaller than $\beta$. Since the operators (id $-\varphi_{\beta}$ ) commute with each other, $\Phi^{\bullet}(D)$ is a chain complex of abelian groups. Note that for each $\alpha \in \Delta$ we have a complex

$$
\Phi_{\alpha}^{\bullet}(D): 0 \rightarrow D^{\stackrel{\text { id }-\varphi_{\alpha}}{\rightarrow}} D \rightarrow 0
$$

such that $\Phi^{\bullet}\left(E_{\Delta}^{s e p}\right)$ is a kind of completed tensor product of the complexes $\Phi_{\alpha}^{\bullet}\left(E_{\alpha}^{s e p}\right)$. More precisely, the tensor product over $\mathbb{F}_{p}$ of the complexes $\Phi^{\bullet}\left(E_{\alpha}^{s e p}\right)$ is the complex $\Phi^{\bullet}\left(E_{\Delta, \circ}^{s e p}\right)$ which is therefore acyclic in nonzero degrees with 0th cohomology equal to $\mathbb{F}_{p}$ by the Künneth formula. Note that there are no higher Tor's as the tensor product is taken over the field $\mathbb{F}_{p}$. We need the following completed version of this observation.

Proposition 4.2. The complex $\Phi^{\bullet}\left(E_{\Delta}^{\text {sep }}\right)$ is acyclic in nonzero degrees with 0th cohomology equal to $\mathbb{F}_{p}$.

The following Lemma is well-known.

Lemma 4.3. For any finite separable extension $E_{\alpha}^{\prime} / E_{\alpha}$ the map id $-\varphi_{\alpha}: X_{\alpha}^{\prime} E_{\alpha}^{\prime+} \rightarrow X_{\alpha}^{\prime} E_{\alpha}^{\prime+}$ is bijective.

Proof. The kernel of id $-\varphi_{\alpha}$ is $\mathbb{F}_{p}$ which is not contained in $X_{\alpha}^{\prime} E_{\alpha}^{\prime+}$. On the other hand, $\sum_{n=0}^{\infty} \varphi_{\alpha}^{n}$ converges on this set and is therefore an inverse to id $-\varphi_{\alpha}$ by formal reasons. 
Our key is the following

Lemma 4.4. For all $\alpha \in S \subseteq \Delta$ the map id $-\varphi_{\alpha}: E_{S}^{\text {sep }} \rightarrow E_{S}^{\text {sep }}$ is surjective with kernel $E_{S \backslash\{\alpha\}}^{s e p} \cdot$

Proof. We may assume $S=\Delta$. The inclusion $E_{\Delta \backslash\{\alpha\}}^{s e p} \subseteq \operatorname{Ker}\left(\mathrm{id}-\varphi_{\alpha}\right)$ is clear. For a collection $E_{\beta} \leq E_{\beta}^{\prime}=\mathbb{F}_{q_{\beta}}\left(\left(X_{\beta}^{\prime}\right)\right)(\beta \in \Delta)$ of finite separable extensions the ring $E_{\Delta}^{\prime}$ is embedded into $\left(E_{\Delta \backslash\{\alpha\}}^{\prime} \otimes_{\mathbb{F}_{p}} \mathbb{F}_{q_{\alpha}}\right)\left(\left(X_{\alpha}^{\prime}\right)\right)$. By comparing the coefficients we find that $\left(E_{\Delta \backslash\{\alpha\}}^{\prime} \otimes_{\mathbb{F}_{p}}\right.$ $\left.\mathbb{F}_{\| \alpha}\right)\left(\left(X_{\alpha}^{\prime}\right)\right)^{\varphi_{\alpha}=\mathrm{id}}=E_{\Delta \backslash\{\alpha\}}^{\prime}$.

For the surjectivity pick an element $c$ in $E_{\Delta}^{\prime} \subset E_{\Delta}^{s e p}$ for some collection of finite separable extensions $E_{\beta} \leq E_{\beta}^{\prime}=\mathbb{F}_{q_{\beta}}\left(\left(X_{\beta}^{\prime}\right)\right)(\beta \in \Delta)$. There exists an integer $k \geq 0$ such that $c$ lies in $X_{\Delta}^{-k} E_{\Delta}^{\prime+}=\widehat{\bigotimes}_{\beta \in \Delta, \mathbb{F}_{p}} X_{\beta}^{-k} E_{\beta}^{\prime+}$. So we may write $c$ as a convergent sum $c=\sum_{n=1}^{\infty} c_{\bar{\alpha}, n} \otimes c_{\alpha, n}$ such that $c_{\bar{\alpha}, n} \in X_{\Delta \backslash\{\alpha\}}^{-k} E_{\Delta \backslash\{\alpha\}}^{\prime+}$ with $c_{\bar{\alpha}, n} \rightarrow 0$ and $c_{\alpha, n} \in X_{\alpha}^{-k} E_{\alpha}^{\prime+}$. Now the images of the elements $c_{\alpha, n}(n \geq 1)$ under the map $E_{\alpha}^{\prime} / X_{\alpha}^{\prime} E_{\alpha}^{\prime+}$ are contained in the finite set $X_{\alpha}^{-k} E_{\alpha}^{\prime+} / X_{\alpha}^{\prime} E_{\alpha}^{\prime+}$, so by Lemma 4.3 there exists an finite separable extension $E_{\alpha}^{\prime} \leq E_{\alpha}^{\prime \prime}$ such that $c_{\alpha, n}=d_{\alpha, n}-\varphi_{\alpha}\left(d_{\alpha, n}\right)$ for some $d_{\alpha, n} \in E_{\alpha}^{\prime \prime}$ for all $n \geq 1$. Moreover, the $X_{\alpha}$-adic valuation of $d_{\alpha, n}$ is bounded by that of the $X_{\alpha}$-adic valuation of $c_{\alpha, n}$ showing that the sum $d:=\sum_{n=1}^{\infty} c_{\bar{\alpha}, n} \otimes d_{\alpha, n}$ defines an element in $E_{\Delta}^{s e p}$ with $c=d-\varphi_{\alpha}(d)$.

Proof of Prop. 4.2. We proceed by induction on $|\Delta|$. The case $|\Delta|=1$ is clear, so suppose $n:=|\Delta|>1$ and we have proven the statement for any proper subset $S \subsetneq \Delta=\left\{\alpha_{1}, \ldots, \alpha_{n}\right\}$. Let $c=\left(c_{S}\right)_{S \in\left(\begin{array}{c}\Delta \\ r\end{array}\right)} \in \bigoplus_{S \in\left(\begin{array}{c}\Delta \\ r\end{array}\right)} E_{\Delta}^{s e p}$ be a cocycle in degree $r$. By Lemma 4.4 we find an element $x=\left(x_{U}\right)_{U \in\left(\begin{array}{c}\Delta \\ r-1\end{array}\right)}$ with $d_{U}=0$ for all $U$ with $\alpha_{n} \nmid U$ such that $\left(c-d^{r-1}(x)\right)_{S}=0$ for all $S \in\left(\begin{array}{c}\Delta \\ r\end{array}\right)$ with $\alpha_{n} \in S$. Indeed, the map $\cup\left\{\alpha_{n}\right\}:\left(\begin{array}{c}\Delta \backslash\left\{\alpha_{n}\right\} \\ r-1\end{array}\right) \rightarrow\left\{S \in\left(\begin{array}{l}\Delta \\ r\end{array}\right) \mid \alpha_{n} \in S\right\}$ is a bijection and by our assumption that $x$ is concentrated into $\left(\begin{array}{c}\Delta \backslash\left\{\alpha_{n}\right\} \\ r-1\end{array}\right) \subset\left(\begin{array}{c}\Delta \\ r-1\end{array}\right)$ only the $S \backslash\{\alpha\}$-component of $x$ contributes to the $S$ component of $d^{r-1}(x)$ for $\alpha_{n} \in S$. So by replacing $c$ with $c-d^{r-1}(x)$ we may assume without loss of generality that $c_{S}=0$ for all $S$ containing $\alpha_{n}$. In particular, for $S^{\prime} \in\left(\begin{array}{c}\Delta \backslash\left\{\alpha_{n}\right\} \\ r\end{array}\right)$ we compute

$$
\begin{array}{r}
0=\left(d^{r}(c)\right)_{S^{\prime} \cup\left\{\alpha_{n}\right\}}=(-1)^{r}\left(\mathrm{id}-\varphi_{\alpha_{n}}\right)\left(c_{S^{\prime}}\right)+\sum_{\beta \in S^{\prime}}(-1)^{\varepsilon(\beta, S)}\left(\mathrm{id}-\varphi_{\beta}\right)\left(c_{S^{\prime} \cup\left\{\alpha_{n}\right\} \backslash\{\beta\}}\right)= \\
=(-1)^{r}\left(\mathrm{id}-\varphi_{\alpha_{n}}\right)\left(c_{S^{\prime}}\right) .
\end{array}
$$

Using Lemma 4.4 again this yields $c_{S^{\prime}} \in E_{\Delta \backslash\left\{\alpha_{n}\right\}}^{\text {sep }}$ for all $S^{\prime} \in\left(\begin{array}{c}\Delta \\ r\end{array}\right)$. Now the statement follows by induction.

The association $D \mapsto \Phi^{\bullet}(D)$ is an exact functor from the category of abelian groups with an action of $\prod_{\alpha \in \Delta} \varphi_{\alpha}^{\mathbb{N}}$ to the category of chain complexes of abelian groups. In particular, for any short exact sequence $0 \rightarrow D_{1} \rightarrow D_{2} \rightarrow D_{3} \rightarrow 0$, we have a short exact sequence $0 \rightarrow \Phi^{\bullet}\left(D_{1}\right) \rightarrow \Phi^{\bullet}\left(D_{2}\right) \rightarrow \Phi^{\bullet}\left(D_{3}\right) \rightarrow 0$ of chain complexes. This yields a long exact sequence

$$
0 \rightarrow h^{0} \Phi^{\bullet}\left(D_{1}\right) \rightarrow h^{0} \Phi^{\bullet}\left(D_{2}\right) \rightarrow h^{0} \Phi^{\bullet}\left(D_{3}\right) \rightarrow h^{1} \Phi^{\bullet}\left(D_{1}\right) \rightarrow h^{1} \Phi^{\bullet}\left(D_{2}\right) \rightarrow h^{1} \Phi^{\bullet}\left(D_{3}\right) \rightarrow \cdots
$$

of abelian groups. 


\subsection{The multivariable $p$-adic coefficient ring}

Our goal in this section is to lift $E_{\Delta}$ and $E_{\Delta}^{s e p}$ to characteristic 0 so we can classify $p$-adic representations of $G_{\mathbb{Q}_{p}, \Delta}$. Recall [5] that $\mathcal{O}_{\mathcal{E}} \cong \lim _{h} \mathbb{Z} /\left(p^{h}\right)((X))$ is constructed as a Cohen ring of $E \cong \mathbb{F}_{p}((X))$. Via the embedding $X \mapsto[\varepsilon]-1$ these are subrings of $\tilde{B}$ which is defined as $\tilde{B}:=W\left(\widehat{E^{s e p}}\right)\left[p^{-1}\right]$ where $W\left(\widehat{E^{s e p}}\right)$ is the ring of $p$-typical Witt vectors of the completion $\widehat{E^{s e p}}$ (with respect to the $X$-adic topology) of the separable closure $E^{\text {sep }}$. Here $[\varepsilon]$ denotes the Teichmüller representative of the sequence $\varepsilon=\left(\varepsilon_{n}\right)_{n} \in \lim _{x \mapsto x^{p}} \mathcal{O}_{\mathbb{C}_{p}} \cong \widehat{E}^{\text {sep }}+$ of $p$-power roots of unity with $\varepsilon_{1} \neq 1$. Note that $\widehat{E^{s e p}}$ is an algebraically closed field of characteristic $p$ which is, in fact, isomorphic to the tilt $\mathbb{C}_{p}^{b}=\operatorname{Frac}\left(\lim _{x x^{p}} \mathcal{O}_{\mathbb{C}_{p}} /(p)\right)$ of $\mathbb{C}_{p}$ in the modern terminology. Further, for any finite extension $E^{\prime} / E$ contained in $E^{s e p}$ there exists a unique finite unramified extension $\mathcal{E}^{\prime}$ of $\mathcal{E}=\mathcal{O}_{\mathcal{E}}\left[p^{-1}\right]$ contained in $\tilde{B}$ with residue field $E^{\prime}$ (Prop. 4.20 in [5]).

We define the ring $\mathcal{O}_{\mathcal{E}_{\Delta}}$ as the projective limit $\lim _{h}\left(\mathbb{Z} /\left(p^{h}\right)\left[\left[X_{\alpha} \mid \alpha \in \Delta\right]\right]\left[X_{\Delta}^{-1}\right]\right)$ and put $\mathcal{E}_{\Delta}:=\mathcal{O}_{\mathcal{E}_{\Delta}}\left[p^{-1}\right]$ so we have $\mathcal{O}_{\mathcal{E}_{\Delta}} /(p) \cong E_{\Delta}$. The Iwasawa algebra $\mathcal{O}_{\mathcal{E}_{\Delta}}^{+}=\mathbb{Z}_{p}\left[\left[X_{\alpha} \mid \alpha \in \Delta\right]\right] \leq$ $\mathcal{O}_{\mathcal{E}_{\Delta}}$ is isomorphic to the completed tensor product of the one-variable Iwasawa algebras $\mathcal{O}_{\mathcal{E}_{\alpha}}^{+}:=\mathbb{Z}_{p}\left[\left[X_{\alpha}\right]\right](\alpha \in \Delta)$ over $\mathbb{Z}_{p}$. This motivates the way we can lift $E_{\Delta}^{\prime}$ to characteristic 0 for a collection $E_{\alpha}^{\prime} / E_{\alpha}(\alpha \in \Delta)$ of finite separable extensions. We define

$$
\mathcal{O}_{\mathcal{E}_{\Delta}^{\prime}}^{+}:=\bigotimes_{\alpha \in \Delta, \mathbb{Z}_{p}} \mathcal{O}_{\mathcal{E}_{\alpha}^{\prime}}
$$

as a completed tensor product. If we write $E_{\alpha}^{\prime}=\mathbb{F}_{q_{\alpha}}\left(\left(X_{\alpha}^{\prime}\right)\right)(\alpha \in \Delta)$ then we may identify $\mathcal{O}_{\mathcal{E}_{\Delta}^{\prime}}^{+}$ with the power series ring $\left(\bigotimes_{\alpha \in \Delta, \mathbb{Z}_{p}} W\left(\mathbb{F}_{q_{\alpha}}\right)\right)\left[\left[X_{\alpha}^{\prime} \mid \alpha \in \Delta\right]\right]$ over the finite étale $\mathbb{Z}_{p}$-algebra $\bigotimes_{\alpha \in \Delta, \mathbb{Z}_{p}} W\left(\mathbb{F}_{q_{\alpha}}\right)$. We define $\mathcal{O}_{\mathcal{E}_{\Delta}^{\prime}}$ as the $p$-adic completion $\widehat{\mathcal{O}_{\mathcal{E}_{\Delta}^{\prime}}^{+}\left[X_{\Delta}^{-1}\right]}=\lim _{h} \mathcal{O}_{\mathcal{E}_{\Delta}^{\prime}}^{+}\left[X_{\Delta}^{-1}\right] /\left(p^{h}\right)$ and put $\mathcal{E}_{\Delta}^{\prime}:=\mathcal{O}_{\mathcal{E}_{\Delta}^{\prime}}\left[p^{-1}\right]$. We have the following alternative characterization of $\mathcal{O}_{\mathcal{E}_{\Delta}^{\prime}}$.

Lemma 4.5. Writing $\Delta=\left\{\alpha_{1}, \ldots, \alpha_{n}\right\}$ we have

$$
\mathcal{O}_{\mathcal{E}_{\Delta}^{\prime}} \cong \mathcal{O}_{\mathcal{E}_{\alpha_{1}}^{\prime}} \otimes_{\mathcal{O}_{\mathcal{E}_{\alpha_{1}}}}\left(\cdots\left(\mathcal{O}_{\mathcal{E}_{\alpha_{n}}^{\prime}} \otimes_{\mathcal{O}_{\mathcal{E}_{\alpha_{n}}}} \mathcal{O}_{\mathcal{E}_{\Delta}}\right)\right)
$$

In particular, $\mathcal{O}_{\mathcal{E}_{\Delta}^{\prime}}$ is a free module of rank $\prod_{i=1}^{n}\left|E_{\alpha_{i}}^{\prime}: E_{\alpha_{i}}\right|$ over $\mathcal{O}_{\mathcal{E}_{\Delta}}$.

Proof. Each $\mathcal{O}_{\mathcal{E}_{\alpha_{i}}^{\prime}}$ is naturally a subring in $\mathcal{O}_{\mathcal{E}_{\Delta}^{\prime}}$ and so is $\mathcal{O}_{\mathcal{E}_{\Delta}}$. Therefore there is a ring homomorphism from the right hand side to the left hand side which is an isomorphism modulo $p$ by Lemma 3.2. The first statement follows from the $p$-adic completeness of both sides.

Since $\mathcal{O}_{\mathcal{E}_{\alpha_{i}}}$ is a complete discrete valuation ring, $\mathcal{O}_{\mathcal{E}_{\alpha_{i}}^{\prime}}$ is finite free over $\mathcal{O}_{\mathcal{E}_{\alpha_{i}}}$ of rank $\left|E_{\alpha_{i}}^{\prime}: E_{\alpha_{i}}\right|(i=1, \ldots, n)$. Therefore the second statement.

Now we define $\mathcal{E}_{\Delta}^{u r}:=\underline{\lim } \mathcal{E}_{\Delta}^{\prime}$ and $\mathcal{O}_{\mathcal{E}_{\Delta}^{u r}}:=\lim _{\longrightarrow} \mathcal{O}_{\mathcal{E}_{\Delta}^{\prime}}$ where $E_{\alpha}^{\prime}$ runs over the finite subextensions of $E_{\alpha}$ in $E_{\alpha}^{\text {sep }}$ for all $\alpha \in \Delta$. Further, we denote by $\widehat{\mathcal{E}_{\Delta}^{u r}}$ (resp. by $\mathcal{O}_{\widehat{\mathcal{E}_{\Delta}^{u r}}}$ ) the p-adic completion of $\mathcal{E}_{\Delta}^{u r}$ (resp. of $\mathcal{O}_{\mathcal{E}_{\Delta}^{u r}}$ ). We have $\mathcal{O}_{\widehat{\mathcal{E}_{\Delta}^{u r}}} /(p) \cong E_{\Delta}^{\text {sep }}$ by construction. The group $G_{\mathbb{Q}_{p}, \Delta}$ acts naturally on $\widehat{\mathcal{E}_{\Delta}^{u r}}$ (resp. on $\mathcal{O}_{\widehat{\mathcal{E}_{\Delta}^{u r}}}$ ). Moreover, for each $\alpha \in \Delta$ we have the Frobenius lift $\varphi_{\alpha}$ on $\tilde{B}_{\alpha}$ (the copy of $\tilde{B}$ indexed by $\alpha$ ) which acts on $[\varepsilon]$ by raising to the $p$ th power 
(as it is a Teichmüller representative). So we have $\varphi_{\alpha}\left(X_{\alpha}\right)=\left(X_{\alpha}+1\right)^{p}-1$. For each finite extension $E_{\alpha}^{\prime} / E_{\alpha}$ we have $\varphi\left(\mathcal{E}_{\alpha}^{\prime}\right) \subset \mathcal{E}_{\alpha}^{\prime}$, so this defines an action of $\varphi_{\alpha}$ on the rings $\mathcal{E}_{\Delta}^{u r}, \mathcal{O}_{\mathcal{E}_{\Delta}^{u r}}$, $\widehat{\mathcal{E}_{\Delta}^{u r}}$, and $\mathcal{O}_{\widehat{\mathcal{E}_{\Delta}}}$ for all $\alpha \in \Delta$. These operators commute with each other and with the action of the group $G_{\mathbb{Q}_{p}, \Delta}$.

Proposition 4.6. We have

$$
\begin{aligned}
& \widehat{\mathcal{E}}_{\Delta}^{u r} H_{\mathbb{Q}_{p}, \Delta}=\mathcal{E}_{\Delta}, \quad \bigcap_{\alpha \in \Delta} \widehat{\mathcal{E}}_{\Delta}^{\varphi^{\varphi_{\alpha}}}=\mathrm{id}=\mathbb{Q}_{p} \text {, and } \\
& \mathcal{O}_{\widehat{\mathcal{E}_{\Delta}^{u r}}}^{H_{\mathbb{Q} p}^{u r}}=\mathcal{O}_{\mathcal{E}_{\Delta}}, \quad \bigcap_{\alpha \in \Delta} \mathcal{O}_{\widehat{\mathcal{E}_{\Delta}^{u r}}}^{\varphi_{\alpha}=\mathrm{id}}=\mathbb{Z}_{p} .
\end{aligned}
$$

Proof. The statements on $\widehat{\mathcal{E}_{\Delta}^{u r}}$ follow from those on $\mathcal{O}_{\widehat{\mathcal{E}_{\Delta}^{u r}}}$ as $p$ is $\varphi_{\alpha^{-}}$and $H_{\mathbb{Q}_{p}, \Delta \text {-invariant for }}$ all $\alpha \in \Delta$. Moreover, the latter statements are consequences of Prop. 3.3, resp. Lemma 3.6 using devissage.

\subsection{The equivalence of categories}

We denote by $\operatorname{Rep}_{\mathbb{Z}_{p}}\left(G_{\mathbb{Q}_{p}, \Delta}\right)$ (resp. by $\left.\operatorname{Rep}_{\mathbb{Q}_{p}}\left(G_{\mathbb{Q}_{p}, \Delta}\right)\right)$ the category of continuous representations of $G_{\mathbb{Q}_{p}, \Delta}$ on finitely generated $\mathbb{Z}_{p}$-modules (resp. on finite dimensional $\mathbb{Q}_{p}$-vector spaces). Let $T$ (resp. $V$ ) be an object in $\operatorname{Rep}_{\mathbb{Z}_{p}}\left(G_{\mathbb{Q}_{p}, \Delta}\right)$ (resp. in $\operatorname{Rep}_{\mathbb{Q}_{p}}\left(G_{\mathbb{Q}_{p}, \Delta}\right)$ ). We define

$$
\mathbb{D}(T):=\left(\mathcal{O}_{\widehat{\mathcal{E}_{\Delta}^{u r}}} \otimes_{\mathbb{Z}_{p}} T\right)^{H_{\mathbb{Q}_{p}, \Delta}} \quad\left(\operatorname{resp.} \mathbb{D}(V):=\left(\widehat{\mathcal{E}_{\Delta}^{u r}} \otimes_{\mathbb{Q}_{p}} V\right)^{H_{\mathbb{Q}_{p}, \Delta}}\right)
$$

By Prop. $4.6 \mathbb{D}(T)$ (resp. $\mathbb{D}(V)$ ) is a module over $\mathcal{O}_{\mathcal{E}_{\Delta}}$ (resp. over $\mathcal{E}_{\Delta}$ ). Moreover, it admits an action of the monoid $T_{+, \Delta}$ : the action of $\varphi_{\alpha}(\alpha \in \Delta)$ is trivial on $T$ (resp. on $V$ ) and therefore comes from the action on $\mathcal{O}_{\widehat{\mathcal{E}_{\Delta}^{u r}}}$ (resp. on $\widehat{\mathcal{E}_{\Delta}^{u r}}$ ) defined above. The action of $\Gamma_{\Delta}=G_{\mathbb{Q}_{p}, \Delta} / H_{\mathbb{Q}_{p}, \Delta}$ comes from the diagonal action of $G_{\mathbb{Q}_{p}, \Delta}$ on $\mathcal{O}_{\widehat{\mathcal{E}_{\Delta}^{u r}}} \otimes_{\mathbb{Z}_{p}} T$ (resp. on $\left.\widehat{\mathcal{E}_{\Delta}^{u r}} \otimes_{\mathbb{Q}_{p}} V\right)$.

Proposition 4.7. Let $T$ be an object in $\operatorname{Rep}_{\mathbb{Z}_{p}}\left(G_{\mathbb{Q}_{p}, \Delta}\right)$. The natural map

$$
\mathcal{O}_{\widehat{\mathcal{E}_{\Delta}^{u r}}} \otimes_{\mathcal{O}_{\mathcal{E}_{\Delta}}} \mathbb{D}(T) \rightarrow \mathcal{O}_{\widehat{\mathcal{E}_{\Delta}^{u r}}} \otimes_{\mathbb{Z}_{p}} T
$$

is an isomorphism.

Proof. This is very similar to the proof of Prop. 2.30 in [5]. We proceed in two steps. Assume first that $T$ is killed by a power $p^{h}$ of $p$. We use induction on $h$. The case $h=1$ is done in Prop. 3.7. Now for $h>1$ we have a short exact sequence $0 \rightarrow T_{1} \rightarrow T \rightarrow T_{2} \rightarrow 0$ of objects in $\operatorname{Rep}_{\mathbb{Z}_{p}}\left(G_{\mathbb{Q}_{p}, \Delta}\right)$ such that $p T_{1}=0$ and $p^{h-1} T_{2}$. Since $\mathcal{O}_{\widehat{\mathcal{E}_{\Delta}^{u r}}}$ has no $p$-torsion, it is flat as $\mathbb{Z}_{p}$-module. Therefore we obtain a short exact sequence

$$
0 \rightarrow \mathcal{O}_{\widehat{\mathcal{E}_{\Delta}^{u r}}} \otimes_{\mathbb{Z}_{p}} T_{1} \rightarrow \mathcal{O}_{\widehat{\mathcal{E}_{\Delta}^{u r}}} \otimes_{\mathbb{Z}_{p}} T \rightarrow \mathcal{O}_{\widehat{\mathcal{E}_{\Delta}^{u r}}} \otimes_{\mathbb{Z}_{p}} T_{2} \rightarrow 0
$$

Now we have an identification $\mathcal{O}_{\widehat{\mathcal{E}_{\Delta}^{u r}}} \otimes_{\mathbb{Z}_{p}} T_{1} \cong E_{\Delta}^{\text {sep }} \otimes_{\mathbb{F}_{p}} T_{1} \cong E_{\Delta}^{\text {sep }} \otimes_{E_{\Delta}} \mathbb{D}\left(T_{1}\right)$. In particular, as a representation of $H_{\mathbb{Q}_{p}, \Delta}$ we have $\mathcal{O}_{\widehat{\mathcal{E}_{\Delta}^{u r}}} \otimes_{\mathbb{Z}_{p}} T_{1} \cong\left(E_{\Delta}^{s e p}\right)^{\operatorname{dim}_{\mathbb{F}_{p}} T_{1}}$. In particular, Prop. 4.1 yields 
$H_{\text {cont }}^{1}\left(H_{\mathbb{Q}_{p}, \Delta}, \mathcal{O}_{\widehat{\mathcal{E}_{\Delta}^{u r}}} \otimes_{\mathbb{Z}_{p}} T_{1}\right)=\{1\}$. By the long exact sequence of continuous $H_{\mathbb{Q}_{p}, \Delta}$-cohomology we deduce the exactness of the sequence

$$
0 \rightarrow \mathbb{D}\left(T_{1}\right) \rightarrow \mathbb{D}(T) \rightarrow \mathbb{D}\left(T_{2}\right) \rightarrow 0
$$

Now we have a commutative diagram

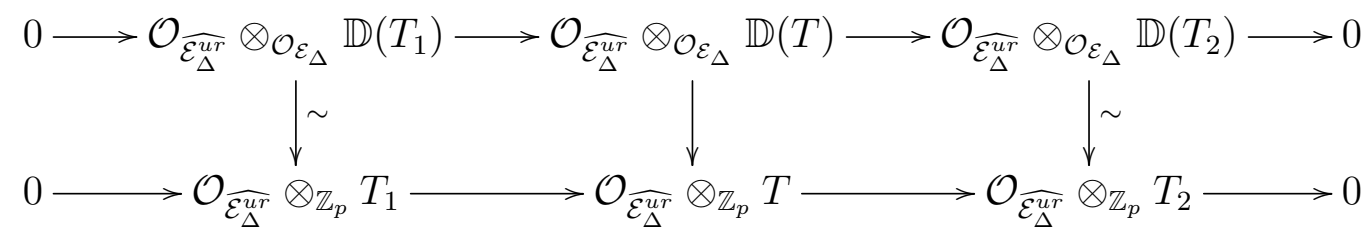

with exact rows. Thus the vertical map in the middle is an isomorphism by induction using the 5-lemma.

The general case follows from this by taking the projective limit of the isomorphisms above for $T / p^{h} T$ as $h$ tends to infinity.

An étale $T_{+, \Delta}$-module over $\mathcal{O}_{\mathcal{E}_{\Delta}}$ is a finitely generated $\mathcal{O}_{\mathcal{E}_{\Delta}}$-module $D$ together with a semilinear action of the monoid $T_{+, \Delta}$ such that for all $\varphi_{t} \in T_{+, \Delta}$ the map

$$
\operatorname{id} \otimes \varphi_{t}: \varphi_{t}^{*} D:=\mathcal{O}_{\mathcal{E}_{\Delta}} \otimes_{\mathcal{O}_{\mathcal{E}_{\Delta}, \varphi_{t}}} D \rightarrow D
$$

is an isomorphism. We denote by $\mathcal{D}^{e t}\left(\varphi_{\Delta}, \Gamma_{\Delta}, \mathcal{O}_{\mathcal{E}_{\Delta}}\right)$ the category of étale $T_{+, \Delta}$-modules over $\mathcal{O}_{\mathcal{E}_{\Delta}}$. As in the mod $p$ case, $\mathcal{D}^{e t}\left(\varphi_{\Delta}, \Gamma_{\Delta}, \mathcal{O}_{\mathcal{E}_{\Delta}}\right)$ has the structure of a neutral Tannakian category. If $D$ is finitely generated $\mathcal{O}_{\mathcal{E}_{\Delta}}$ module that is killed by a power $p^{h}$ of $p$ we define the generic length of $D$ as length ${ }_{g e n} D:=\sum_{i=1}^{h} \operatorname{rk}_{E_{\Delta}} p^{i-1} D / p^{i} D$ where $\operatorname{rk}_{E_{\Delta}}$ denotes the generic rank (ie. dimension over $\operatorname{Frac}\left(E_{\Delta}\right)$ of the localisation at $\left.(0)\right)$.

Corollary 4.8. The functor $\mathbb{D}$ is exact. $\mathbb{D}(T)$ is an object in $\mathcal{D}^{\text {et }}\left(\varphi_{\Delta}, \Gamma_{\Delta}, \mathcal{O}_{\mathcal{E}_{\Delta}}\right)$ for any $T$ in $\operatorname{Rep}_{\mathbb{Z}_{p}}\left(G_{\mathbb{Q}_{p}, \Delta}\right)$. Moreover, if $T$ is killed by a power of $p$ then the we have length ${ }_{\text {gen }} \mathbb{D}(T)=$ length $_{\mathbb{Z}_{p}} T$.

Proof. If $T$ is an object in $\operatorname{Rep}_{\mathbb{Z}_{p}}\left(G_{\mathbb{Q}_{p}, \Delta}\right)$ such that $p^{h} T=0$, then we have $H^{1}\left(H_{\mathbb{Q}_{p}, \Delta}, \mathcal{O}_{\widehat{\mathcal{E}_{\Delta}^{u r}}} \otimes_{\mathbb{Z}_{p}}\right.$ $T)=\{1\}$ by induction on $h$ using the long exact sequence of continuous $H_{\mathbb{Q}_{p}, \Delta}$-cohomology. So the exactness of $\mathbb{D}$ on finite length objects in $\operatorname{Rep}_{\mathbb{Z}_{p}}\left(G_{\mathbb{Q}_{p}, \Delta}\right)$ follows the same way as in the proof of Prop. 4.7 in the special case when $p T_{1}=0$. Now if $0 \rightarrow T_{1} \rightarrow T_{2} \rightarrow T_{3} \rightarrow 0$ is an arbitrary short exact sequence in $\operatorname{Rep}_{\mathbb{Z}_{p}}\left(G_{\mathbb{Q}_{p}, \Delta}\right)$ then we have an exact sequence

$$
0 \rightarrow T_{1}\left[p^{h}\right] \rightarrow T_{2}\left[p^{h}\right] \rightarrow T_{3}\left[p^{h}\right] \stackrel{\partial_{h}}{\rightarrow} T_{1} / p^{h} T_{1} \rightarrow T_{2} / p^{h} T_{2} \rightarrow T_{3} / p^{h} T_{3} \rightarrow 0
$$

of finite length objects for all $h \geq 1$. Applying $\mathbb{D}$ yields an exact sequence

$$
0 \rightarrow \mathbb{D}\left(T_{1}\left[p^{h}\right]\right) \rightarrow \mathbb{D}\left(T_{2}\left[p^{h}\right]\right) \rightarrow \mathbb{D}\left(T_{3}\left[p^{h}\right]\right) \rightarrow \mathbb{D}\left(T_{1} / p^{h} T_{1}\right) \rightarrow \mathbb{D}\left(T_{2} / p^{h} T_{2}\right) \rightarrow \mathbb{D}\left(T_{3} / p^{h} T_{3}\right) \rightarrow 0
$$

for all $h \geq 1$. Since $T_{i}$ is finitely generated over $\mathbb{Z}_{p}$, we have $T_{i}\left[p^{h}\right]=\left(T_{i}\right)_{\text {tors }}$ for $h \geq h_{0}$ large enough $(i=1,2,3)$. In particular, the connecting map $T_{i}\left[p^{(n+1) h}\right] \stackrel{p^{h}}{\rightarrow} T_{i}\left[p^{n h}\right]$ is the zero map for $h \geq h_{0}$ and $i=1,2,3$. Thus the Mittag-Leffler property is satisfied for both $\operatorname{Im}\left(\partial_{h}\right)_{h}$ and 
Coker $\left(\partial_{h}\right)_{h}$ as the map $T_{1} / p^{h+1} T_{1} \rightarrow T_{1} / p^{h} T_{1}$ is surjective for all $h \geq 1$. Hence taking the projective limit we obtain an exact sequence $0 \rightarrow \mathbb{D}\left(T_{1}\right) \rightarrow \mathbb{D}\left(T_{2}\right) \rightarrow \mathbb{D}\left(T_{3}\right) \rightarrow 0$ as claimed.

The statement on the generic length follows from the exactness using Prop. 3.7 and induction on $h$ such that $p^{h} T=0$. In particular, $\mathbb{D}(T)$ is finitely generated over $\mathcal{O}_{\mathcal{E}_{\Delta}}$ if $T$ has finite length. Now if $T$ is not necessarily of finite length then we apply the exactness of $\mathbb{D}$ on the exact sequence $0 \rightarrow T[p] \rightarrow T \stackrel{p \cdot}{\rightarrow} T \rightarrow T / p T \rightarrow 0$ we obtain that $\mathbb{D}(T / p T)=\mathbb{D}(T) / p \mathbb{D}(T)$ which is finitely generated over $E_{\Delta}$. Therefore $\mathbb{D}(T)$ is finitely generated over $\mathcal{O}_{\mathcal{E}_{\Delta}}$ by the $p$-adic completeness of $\mathbb{D}(T)$ (by definition we have $\lim _{h} \mathbb{D}\left(T / p^{h} T\right)=\mathbb{D}(T)$ ).

Finally, the étale property for finite length modules follows by induction on the length from the case $h=1$ (Prop. 3.7) and in general by taking the projective limit.

Conversely, let $D$ be an object in $\mathcal{D}^{e t}\left(\varphi_{\Delta}, \Gamma_{\Delta}, \mathcal{O}_{\mathcal{E}_{\Delta}}\right)$. We define

$$
\mathbb{T}(D):=\bigcap_{\alpha \in \Delta}\left(\mathcal{O}_{\widehat{\mathcal{E}_{\Delta}^{u r}}} \otimes_{\mathcal{O}_{\mathcal{E}_{\Delta}}} D\right)^{\varphi_{\alpha}=\mathrm{id}}
$$

This is a $\mathbb{Z}_{p}$-module admitting a diagonal action of $G_{\mathbb{Q}_{p}, \Delta}$ via the formula $g(\lambda \otimes d):=g(\lambda) \otimes$ $\chi(g)(d)$ where $\chi: G_{\mathbb{Q}_{p}, \Delta} \rightarrow \Gamma_{\Delta}$ is the quotient map.

Proposition 4.9. For any object $D$ in $\mathcal{D}^{e t}\left(\varphi_{\Delta}, \Gamma_{\Delta}, \mathcal{O}_{\mathcal{E}_{\Delta}}\right)$, the natural map

$$
\mathcal{O}_{\widehat{\mathcal{E}_{\Delta}^{u r}}} \otimes_{\mathbb{Z}_{p}} \mathbb{T}(D) \rightarrow \mathcal{O}_{\widehat{\mathcal{E}_{\Delta}^{u r}}} \otimes_{\mathcal{O}_{\mathcal{E}_{\Delta}}} D
$$

is an isomorphism.

Proof. This is completely analogous to the proof of Prop. 2.31 in [5]. We proceed in two steps. At first assume that $p^{h} D=0$ for some integer $h \geq 1$. Consider the exact sequence $0 \rightarrow D[p] \rightarrow D \rightarrow D / D[p] \rightarrow 0$ and apply the exact functor $\Phi^{\bullet} \circ\left(\mathcal{O}_{\widehat{\mathcal{E}_{\Delta}^{u r}}} \otimes_{\mathcal{O}_{\mathcal{E}_{\Delta}}} \cdot\right)$ to obtain an exact sequence

$$
0 \rightarrow \Phi^{\bullet}\left(\mathcal{O}_{\widehat{\mathcal{E}_{\Delta}^{u r}}} \otimes_{\mathcal{O}_{\mathcal{E}_{\Delta}}} D[p]\right) \rightarrow \Phi^{\bullet}\left(\mathcal{O}_{\widehat{\mathcal{E}_{\Delta}^{u r}}} \otimes_{\mathcal{O}_{\mathcal{E}_{\Delta}}} D\right) \rightarrow \Phi^{\bullet}\left(\mathcal{O}_{\widehat{\mathcal{E}_{\Delta}^{u r}}} \otimes_{\mathcal{O}_{\mathcal{E}_{\Delta}}} D / D[p]\right) \rightarrow 0
$$

By Thm. $3.15 D[p]$ is in the image of the functor $\mathbb{D}$ whence $\mathcal{O}_{\widehat{\mathcal{E}_{\Delta}^{u r}}} \otimes_{\mathcal{O}_{\mathcal{E}_{\Delta}}} D[p]$ is isomorphic to $\left(E_{\Delta}^{s e p}\right)^{\mathrm{rk}_{E_{\Delta}} D[p]}$ as a $\prod_{\alpha \in \Delta} \varphi_{\alpha}^{\mathbb{N}}$-module using Prop. 3.7. In particular, $h^{1} \Phi^{\bullet}\left(\mathcal{O}_{\widehat{\mathcal{E}_{\Delta}^{u r}}} \otimes_{\mathcal{O}_{\mathcal{E}_{\Delta}}} D[p]\right)=0$ by Prop. 4.2. This yields an exact sequence

$$
0 \rightarrow \mathbb{T}(D[p]) \rightarrow \mathbb{T}(D) \rightarrow \mathbb{T}(D / D[p]) \rightarrow 0
$$

and the statement follows the same way as in the proof of Prop. 4.7 .

The general case follows by taking the limit.

Now note that $\mathbb{T}(D)$ is finitely generated over $\mathbb{Z}_{p}$ : this is obvious in the case when $p^{h} D=0$ using induction on $h$ and in the general case by Nakayama's lemma as we have $\mathbb{T}(D)=$ $\lim _{h} \mathbb{T}\left(D / p^{h} D\right)$ by construction. So we deduce

Theorem 4.10. The functors $\mathbb{D}$ and $\mathbb{T}$ are quasi-inverse equivalences of categories between the Tannakian categories $\operatorname{Rep}_{\mathbb{Z}_{p}}\left(G_{\mathbb{Q}_{p}, \Delta}\right)$ and $\mathcal{D}^{e t}\left(\varphi_{\Delta}, \Gamma_{\Delta}, \mathcal{O}_{\mathcal{E}_{\Delta}}\right)$. 
Finally, an étale $T_{+, \Delta}$-module over $\mathcal{E}_{\Delta}$ is a finitely generated $\mathcal{E}_{\Delta}$-module $D$ together with a semilinear action of the monoid $T_{+, \Delta}$ such that there exists an object $D_{0}$ in $\mathcal{D}^{e t}\left(\varphi_{\Delta}, \Gamma_{\Delta}, \mathcal{O}_{\mathcal{E}_{\Delta}}\right)$ with an isomorphism $D \cong D_{0}\left[p^{-1}\right]=\mathcal{E}_{\Delta} \otimes_{\mathcal{O}_{\mathcal{E}}} D_{0}$. We denote by $\mathcal{D}^{e t}\left(\varphi_{\Delta}, \Gamma_{\Delta}, \mathcal{E}_{\Delta}\right)$ the category of étale $T_{+, \Delta}$-modules over $\mathcal{E}_{\Delta}$. As before, $\mathcal{D}^{\text {et }}\left(\varphi_{\Delta}, \Gamma_{\Delta}, \mathcal{E}_{\Delta}\right)$ has the structure of a neutral Tannakian category. We have the following characteristic 0 version of the category equivalence:

Theorem 4.11. The functors

$$
\begin{aligned}
V & \mapsto \mathbb{D}(V):=\left(\widehat{\mathcal{E}_{\Delta}^{u r}} \otimes_{\mathbb{Q}_{p}} V\right)^{H_{\mathbb{Q}_{p}, \Delta}} \\
D & \mapsto \mathbb{V}(D):=\bigcap_{\alpha \in \Delta}\left(\widehat{\mathcal{E}_{\Delta}^{u r}} \otimes_{\mathcal{E}_{\Delta}} D\right)^{\varphi_{\alpha}=\mathrm{id}}
\end{aligned}
$$

are quasi-inverse equivalences of categories between the Tannakian categories $\operatorname{Rep}_{\mathbb{Q}_{p}}\left(G_{\mathbb{Q}_{p}, \Delta}\right)$ and $\mathcal{D}^{e t}\left(\varphi_{\Delta}, \Gamma_{\Delta}, \mathcal{E}_{\Delta}\right)$.

Proof. Since $G_{\mathbb{Q}_{p}, \Delta}$ is compact, any finite dimensional $\mathbb{Q}_{p}$-representation $V$ contains a $G_{\mathbb{Q}_{p}, \Delta^{-}}$ invariant lattice $T$. The statement follows from Thm. 4.10 by inverting $p$ on both sides. The compatibility with tensor products and duals follows the same way as in characteristic $p$.

Remarks. 1. If $A$ is a $\mathbb{Z}_{p}$-algebra which is finitely generated as a module over $\mathbb{Z}_{p}$, then we have an equivalence of categories between $\operatorname{Rep}_{A}\left(G_{\mathbb{Q}_{p}, \Delta}\right)$ and $\mathcal{D}^{e t}\left(\varphi_{\Delta}, \Gamma_{\Delta}, A \otimes_{\mathbb{Z}_{p}} \mathcal{O}_{\mathcal{E}_{\Delta}}\right)$. Indeed, we have a natural isomorphism $\left(A \otimes_{\mathbb{Z}_{p}} \mathcal{O}_{\widehat{\mathcal{E}_{\Delta}^{u r}}}\right) \otimes_{A} \cong \mathcal{O}_{\widehat{\mathcal{E}_{\Delta}^{u r}}} \otimes_{\mathbb{Z}_{p}} \cdot$ as functors on $\operatorname{Rep}_{A}\left(G_{\mathbb{Q}_{p}, \Delta}\right)$. Similarly, if $K$ is a finite extension of $\mathbb{Q}_{p}$, then we have an equivalence of categories between $\operatorname{Rep}_{K}\left(G_{\mathbb{Q}_{p}, \Delta}\right)$ and $\mathcal{D}^{e t}\left(\varphi_{\Delta}, \Gamma_{\Delta}, K \otimes_{\mathbb{Q}_{p}} \mathcal{E}_{\Delta}\right)$.

2. It is expected that there is a similar equivalence of categories for representations of the $|\Delta|$ th direct power of the group $\operatorname{Gal}\left(\overline{\mathbb{Q}_{p}} / F\right)$ for a finite extension $F / \mathbb{Q}_{p}$. However, at this point it is not clear what type of $(\varphi, \Gamma)$-modules one should consider. The usual cyclotomic $(\varphi, \Gamma)$-modules do not seem to be well-suited for the purpose of the $p$-adic and $\bmod p$ Langlands programme. On the other hand, the Lubin-Tate setting may not work properly in characteristic $p$ due to the non-existence of the distinguished left inverse $\psi$ of $\varphi$. To work over the character variety of the group $\mathcal{O}_{F}$ [2] seems, however, to be a good candidate.

\section{References}

[1] Balister P., Howson S., Note on Nakayama's lemma for compact $\Lambda$-modules, Asian $J$. Math. 1(2) (1997), 224-229.

[2] Berger L., Schneider P., Xie B., Rigid character groups, Lubin-Tate theory, and $(\varphi, \Gamma)$ modules, preprint (2015)

[3] Colmez P., $(\varphi, \Gamma)$-modules et représentations du mirabolique de $G L_{2}\left(\mathbb{Q}_{p}\right)$, Astérisque 330 (2010), 61-153.

[4] Dee J., $\Phi-\Gamma$-Modules for families of Galois representations, J. of Algebra 235 (2001), 636-664. 
[5] Fontaine J.-M., Ouyang Y., Theory of $p$-adic Galois representations, book in preparation

[6] Hartshorne R., Residues and duality, Springer (1966).

[7] Scholze P., Lecture notes on p-adic geometry (written by J. Weinstein), https://math.berkeley.edu/ jared/Math274/ScholzeLectures.pdf

[8] Wedhorn Th., Adic spaces, preprint (2012)

[9] Weibel Ch., An introduction to homological algebra, Cambridge studies in advanced mathematics 38, Cambridge University Press, 1994.

[10] Weibel Ch., The K-book: An introduction to algebraic K-theory, Graduate Studies in Math. vol. 145, AMS, 2013.

[11] Zábrádi G., Multivariable $(\varphi, \Gamma)$-modules and smooth $o$-torsion representations, preprint (2015), arXiv: 1511.01037 